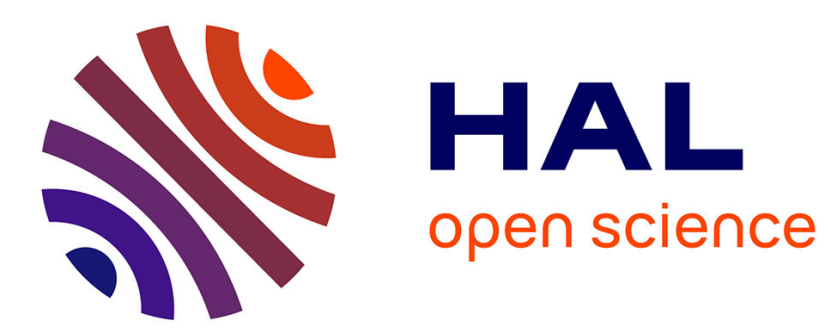

\title{
Les marches urbaines exploratoires de nuit: une critique socio-urbaine en situation
}

Florian Guérin, Edna Hernández González

\section{To cite this version:}

Florian Guérin, Edna Hernández González. Les marches urbaines exploratoires de nuit : une critique socio-urbaine en situation. Sciences du Design, 2017, Design et santé, 2 (6), pp.105-127. hal-01393575

\section{HAL Id: hal-01393575 \\ https://hal.science/hal-01393575}

Submitted on 7 Nov 2016

HAL is a multi-disciplinary open access archive for the deposit and dissemination of scientific research documents, whether they are published or not. The documents may come from teaching and research institutions in France or abroad, or from public or private research centers.
L'archive ouverte pluridisciplinaire HAL, est destinée au dépôt et à la diffusion de documents scientifiques de niveau recherche, publiés ou non, émanant des établissements d'enseignement et de recherche français ou étrangers, des laboratoires publics ou privés. 


\title{
Les marches urbaines exploratoires de nuit : une critique urbaine en situation
}

\author{
Florian Guérin \\ Doctorant à l'Université Paris-Est, France \\ Lab'Urba (EA 3482), Urbanisme et aménagement, GT MUP (Labex FutursUrbains) \\ guerinflo@gmail.com
}

Edna Hernández González

Maître de conférences à l'Université de Bretagne Occidentale, France

Institut de Géoarchitecture (EA 2219), Architecture et Urbanisme, GT MUP (Labex FutursUrbains)

andemsl@yahoo.fr

\begin{abstract}
RÉSUMÉ
Dans cet article, nous voulons solidifier l'hypothèse selon laquelle le renouvellement de la critique urbaine systématisée de la société contemporaine est parallèle au développement des travaux sur la marche urbaine comprise comme « fait social total » (M. Mauss, 1923-24). La marche urbaine, en tant qu'expérimentation méthodologiquement cadrée, permettrait un regard in situ sur le monde urbain, amenant à une réflexivité critique sur l'aménagement urbain (dont sa gestion et le discours à propos), face au vécu, au subversif et aux sens (ontologique et perceptifs) de ses usagers. Il nous faudra démontrer que cette articulation fructueuse permet une compréhension complexe (E. Morin, 1990) de l'urbanité nocturne occidentale, en réinterrogeant les catégories analytiques usuelles. La méthodologie des Marches urbaines exploratoires de nuit est basée sur un large corpus comprenant les disgnoastics urbains (en marchant, explorations de M. Armengaud et L. Gwiazdzinski,...), les critiques du mouvement moderne (G. Debord et ses dérives urbaines, JF Augoyard,...) et les explorations urbaines sensibles (démarches de JP Thibaud, JY Petiteau, cartes mentales,...).
\end{abstract}

MOTS-CLÉS : vie nocturne, espace urbain, méthodologie qualitative, marche urbaine

\begin{abstract}
The article deals with the hypothesis of a relationship between the new systematized urban criticism and the development of scientific literature on urban walking as a global social fact (M. Mauss, 1923-24). The methodologically experimentation of urban walking would allow an in situ comprehension of urban world: a critic about urban planning (how it is managed and the discursive around) faced to how it's used and lived. We have to prove that articulation is successful to a complex comprehension (E. Morin, 1990) of Western nocturnal urbanity. This is a way to question usual analytic categories linked to a situation. Thus, we experimented a new methodology: exploratory urban night-walks. They are based on a wide corpus like urban diagnosis (diagnosis by walking, explorations of M. Armengaud and L. Gwiazdzinski, ...), critics of modern movement (G. Debord and urban drifts, JF Augoyard, ...) and sensitive and urban explorations (demarches of JP Thibaud, JY Petiteau, cognitive map, ...).
\end{abstract}

KEYWORDS: night-life, urban spaces, qualitative methodology, urban walking 
« Chacun ne peut voir qu'à sa lampe ;

mais il peut marcher ou agir à la lumière d'autrui », J. Joubert (1866) ${ }^{1}$

\section{Introduction}

Cet ancien secrétaire de Diderot, ayant rédigé nombre de lettres à vocation moraliste et à propos de la nature de l'Homme, nous permet d'introduire notre réflexion sur l'articulation entre la marche urbaine et la marche intellectuelle. En effet, les nuits obscures de la ville et de la connaissance sont inaccessibles directement à l'Homme. Impénétrables, il ne pourra que tenter de se référer à sa perception déformée d'un monde sensible. Ces illusions nécessitent un médium et une méthode pour construire son cheminement, soit un éclairage. Cet éclairage peut être physique (l'éclairage urbain, la lampe torche, etc.) et, surtout, intellectuel (les écrits d'autres auteurs, la pensée ne pouvant pas se construire ex nihilo). Ces médiums permettent à l'Homme d'avancer dans la ville et dans ses pensées, de parvenir au monde des Idées débarrassé de l'illusion des opinions (Platon, «Allégorie de la caverne »). Cependant, cette marche n'est pas aisée, elle demande des efforts physiques et intellectuels pour s'extirper de ce monde d'illusions et conduire la pensée.

Notre proposition est d'amener le chercheur à articuler la marche (au sens physique) à la dé-marche (au sens intellectuel) pour permettre une connaissance du monde urbain nocturne. Le monde de la nuit étant emplit de significations mythico-religieuses, vécu souvent sous un mode sensible voire irrationnel, il nous semblait important de s'y extirper (non de s'en échapper) pour arriver à une compréhension scientifique des nuits urbaines. Nous verrons que ceci ne peut se produire qu'au prisme d'efforts physiques, en suivant la lumière de la ville, et grâce à l'éclairage d'un corpus scientifique solide, d'autrui. Néanmoins, cette compréhension analytique ne peut être similaire à la structuration diurne de la science et de la marche car la nuit révélerait «d'autres manières d'être ensemble au monde » (E. Heurgon, 2005). Il semble donc nécessaire de se détourner des éclairages illusoires d'une perspective diurne, de préconceptions scientifiques d'un monde autre. Ceci implique la nécessité d'outils pour penser le monde nocturne, notamment urbain, c'est-à-dire, les relations spécifiques entre cet espace-temps, le milieu non-humain et l'Homme (tel que les anthropologues A. Monod Becquelin et J. Galinier nous invitent à effectuer).

Pour cela, nous partons de l'hypothèse que la marche, en tant qu'expérimentation méthodologiquement cadrée, permettrait le renouvellement de la critique urbaine systématisée en un espace-temps nocturne. Cette démarche méthodologique en marchant permettrait un regard in situ sur le monde urbain, une réflexivité critique articulant : la manière dont un espace-temps urbain est pensé au niveau de son aménagement ; conçu concrètement et promu ; géré et utilisé/vécu ; perçu (aux sens ontologique et sensible) par les usagers, en fonction de leurs attentes initiales. Il s'agit donc d'identifier les interactions entre la conception et l'aménagement

${ }^{1}$ JOUBERT, J. (1866), « De la sagesse », XLI 
des espaces-temps urbains, leur gestion, les usages et non-usages de ces espacestemps et le discours sur ces espaces-temps, pour leur promotion et à propos de leur perception. Il nous faudra démontrer que cette articulation fructueuse permet une compréhension complexe (E. Morin, 1990) de l'urbanité nocturne occidentale, en réinterrogeant les catégories analytiques usuelles - une réinterrogation dynamique liée à une situation (une configuration espace-tempsaction) et en prise avec cette situation.

Afin de mettre à l'épreuve cette hypothèse - construite de manière inductive suite à une enquête de terrain dans le cadre d'une thèse de doctorat de chacun des auteurs $^{2}$ - nous avons tenté de mettre en place une méthodologie de marches urbaines exploratoires, c'est-à-dire, de traversées collectives nocturnes d'une diversité morphologique aux niveaux spatial, temporel et social. La ville de Paris a été choisie comme terrain d'étude, du fait de la connaissance des problématiques locales aux niveaux de la marchabilité ${ }^{3}$ et de la nuit urbaine ${ }^{4}$; une connaissance ethnographique préalable et nécessaire au bien-fondé de la méthodologie.

Trois événements nous ont permis de proposer cette exploration à 62 participants, aux profils sociaux et de marcheurs nocturnes variés, afin de caractériser les nuits urbaines parisiennes, d'avoir une réflexivité critique sur la méthodologie et de renouveler les démarches compréhensives. Nous pourrons ainsi dévoiler les types de résultats empiriques et réorientations méthodologiques de ces expérimentations, principalement basés sur l'élaboration de carnets d'écriture, de cartes mentales, de photographies et d'observations systématisées et guidées. Ces résultats nous amèneront à discuter les limites de l'articulation entre la marche (en tant que méthodologie d'analyse urbaine, moyen de la participation citoyenne et mode de déplacement spécifique) et la critique urbaine et sociale, en situation spécifique; soit une articulation dynamique entre la compréhension micro- d'une situation socio-urbaine et son impact macro- à l'échelle sociétale.

\section{Les innovations méthodologiques des marches urbaines exploratoires pour comprendre les nuits urbaines :}

En nous appuyant sur des méthodologies d'analyse sensible du territoire, nous allons définir la méthodologie des marches urbaines exploratoires que nous avons mise en place, ses contours et apports. Nous verrons alors les enjeux heuristiques de l'articulation entre, ce que nous appelons, une chrono-cartographie sensible et un diagnostic urbain des nuits parisiennes, soit une articulation entre une optique objective et subjective.

\footnotetext{
${ }^{2}$ GUERIN, F. (en cours), «Le noctambulisme à Paris et Madrid. Les propriétés situationnelles en milieu festif face aux formes de régulation institutionnelle des pratiques de publicisation de configurations sociourbaines nocturnes », thèse de doctorat en Urbanisme et aménagement, dir. J. Monnet, Université Paris-Est ; HERNANDEZ GONZALEZ, E. (2010), Université Paris-Est

${ }^{3}$ Retour de la figure du «piéton » en hyper-centre urbain, cohabitations entre les diverses formes de mobilité, etc., via le groupe thématique « Mobilités Urbaines Pédestres », Labex FutursUrbains

${ }^{4}$ Formes de cohabitations et de conceptions divergentes de la nuit, etc., via les recherches des auteurs
} 


\section{1. État de l'art des explorations urbaines :}

L'espace-temps nocturne a été longtemps oublié par les réflexions sur le fonctionnement urbain, le développement des territoires et la gestion dans les usages des espaces urbains. Alors que les artistes l'avaient investi depuis bien longtemps (L. Gwiazdzinski, 2005), les aménageurs et chercheurs ne l'ont considéré qu'à partir des années 2000-2005, malgré le travail pionnier d'A. Cauquelin datant de 1977. En effet, les travaux suivant ont surtout porté sur l'éclairage nocturne, où le vocabulaire (autour de «la fée électrique ») rappelle le côté «magique » de cette technique ${ }^{5}$ et l'objet rappelle l'intérêt artistique du clairobscur $^{6}$, dont l'articulation permet de dessiner les nuits urbaines via des jeux d'obscurité.

Afin d'articuler les premiers savoirs sur les nuits urbaines contemporaines à l'action publique, une technique d'explorations urbaines a été mise en place par M. Armengaud et L. Gwiazdzinski dès les années 1990. L'objectif était davantage politique que scientifique : interpeler les pouvoirs publics sur l'importance d'une temporalité nocturne délaissée, les faire dialoguer avec d'autres types d'acteurs (artistes, urbanistes, chercheurs, citoyens, etc.) et envisager des innovations en termes d'aménagement urbain afin de permettre une hospitalité urbaine. Ces traversées étaient clairement envisagées sous un prisme sensible, une sorte de relevé systématique des sensations et impressions procurées par les lieux traversés (L. Gwiazdzinski, 2006). Malgré tout l'intérêt de cette démarche, il nous semblait que la construction des outils possédait quelques lacunes. En effet, les « interviews d'habitant-e de la nuit» étaient construites à partir de pseudoquestions larges, déconnectées de l'univers symbolique des interrogés et menant à la construction superficielle de dichotomies et catégorisations non forcément partagées. Ainsi, les résultats étaient de faible portée et généraux, et les participants étaient encouragés à livrer des pistes d'action dès le lendemain, sans analyse préalable des données recueillies. On peut donc se demander s'il ne s'agit pas plutôt de faire valider des catégorisations préconstruites que d'analyser les nuits urbaines spécifiques à chaque contexte.

Ainsi, d'autres méthodes ont été développées et éprouvées pour analyser les effets sensitifs d'un milieu sur l'individu, et inversement. Initiées au CRESSON, elles consistent en une mise en récit du parcours en temps réel, ainsi que des interactions avec le milieu et ses «prises». La méthode des «parcours commentés » de JP Thibaud (2001) permet d'analyser un espace urbain en évitant le biais des caractéristiques individuelles de l'observateur (qui débute par décrire sa propre expérience sensible). La «méthode des itinéraires» (JY Petiteau, E. Pasquier, 2001) est proche. Il s'agit, pour le participant, d'énoncer les souvenirs et ressentis provoqués par la réalisation du parcours (donc qui retrouvent au présent une évidence première). Ces techniques d'enquête permettent d'avoir accès aux micro-rituels du quotidien et de mettre en évidence le caractère contextuel des conduites sociales. Néanmoins, il peut sembler artificiel de verbaliser le processus

\footnotetext{
${ }^{5}$ Le vocabulaire se spécifiera par la suite, notamment autour de la professionnalisation de nouveaux acteurs, tels que les «urbanistes lumière »

${ }^{6}$ Une technique de peinture à l'huile apparue dès la Renaissance en Occident
} 
menant à prendre des microdécisions parfois non conscientes et une «fracture sociale » est visible entre les participants ayant la compétence de décrire leurs ressentis ou non. Ainsi, il est possible de retrouver des prénotions portant sur le lieu et inculquées par le participant mais ne lui correspondant pas. Se pose également la question de l'habitude, la routine, qui rend le parcours « intime» pour le participant le pratiquant quotidiennement et change son rapport au lieu, par rapport à un Etranger au sens large.

Il semble que ces développements fassent suite aux critiques du mouvement moderne, notamment à la formalisation des «dérives urbaines » de Guy Debord. Il s'agit d'effectuer une carte des reliefs «psychogréographiques », c'est-à-dire, du rôle des coupures du tissu urbain sur la réalisation d'un parcours qui se veut aléatoire, mais, est dirigé par l'habitude de l'individu et sa perception de l'ambiance urbaine du milieu traversé (donc des sollicitations du terrain). L'objectif est de modifier les pratiques urbanistiques, afin de supprimer les marges interstitielles, soit une critique des espaces réels, représentés et vécus. Néanmoins, ces dérives ne semblent pas pouvoir s'effectuer de nuit, la fatigue amenant à l'abandon (GE Debord, 1958).

Ces méthodologies d'explorations urbaines sont de véritables critiques de la société moderne. Elles permettent une compréhension du monde contemporain à partir de l'analyse de ses continuités/discontinuités historiques d'avec la société moderne, en termes politiques, technico-économiques et identitaires. Il s'agit d'analyser les ruptures dans l'organisation des rapports sociaux entre la modernité « classique » occidentale et le temps présent. L'aménagement urbain - jouant un rôle sur les comportements humains - en serait le principal marqueur. L'aménagement de l'espace produit des effets sur la forme sociale, la mise en scène des groupes sociaux. Mais, ces espaces sont également sans cesse réappropriés, de manière éphémère, par l'affirmation des besoins propres à une population. Ainsi, l'espace est l'ensemble des relations qui unissent les individus à leur environnement et qui fondent l'identité du groupe. Il est symbolisé, chargé de sens. Il s'agit d'un " habiter », d'un espace "vécu » et "représenté », soit un espace différencié. Or, cette différenciation, ce «vécu », semble problématique au niveau de critères d'hospitalité urbaine, notamment de nuit. Ainsi, les explorations urbaines ont tenté de mettre à jour ces problématiques pour envisager une autre ville. Pouvant être perçus comme subversifs, à contre-courant, ces explorations ont permis une reconfiguration de la perception sociale des phénomènes étudiés, en réinterrogeant les catégories analytiques usuelles (en sciences sociales et dans le langage commun), via des moyens innovants et peu communs.

Fondées sur le «sensible», elles pourraient néanmoins être comprises comme illusoires, en décalage avec le réel, relatives au monde des opinions. Ce serait omettre le fait que ces situations problématiques sont issues d'une pensée de la modernité se voulant scientiste, prétendument objective. Via la démarche hypothético-déductive (d'après la vision positiviste de C. Bernard), le chercheur se considère extérieur à son objet d'analyse afin de garantir une objectivité et une scientificité de sa démarche. Cette croyance des sciences humaines et sociales, basée sur une certaine idée de la démarche en sciences naturelles, est largement 
remise en cause aujourd'hui. H. Arendt défend l'idée selon laquelle la science moderne serait entrée en crise suite au rejet de la connaissance sensible au profit des connaissances rationnelles, par la «mathématisation de la physique » $(\mathrm{H}$. Arendt, 1958: 360-361). Du coup, le prisme d'un structuralisme ou fonctionnalisme clos et enfermant ne permet pas de rendre compte du sens de l'engagement des individus dans une action, de leur rapport à un milieu urbain qui influence leurs comportements et est produit, en retour, par leurs formes d'occupation et de cohabitation. La compréhension de ces univers de signification, et de leurs interactions avec le milieu (social, urbain, etc.) dans lequel ils prennent place, ne peut se faire que par une articulation entre objectivité et subjectivité. Ces deux dimensions se trouvent en relation dialogique et il serait vain de vouloir se passer de l'une ou de l'autre dans le travail scientifique. L'émotion ressentie permet d'agir dans un milieu et de le comprendre, de lui donner du sens. Or, le monde social se transforme continuellement. Donc, cette articulation est dynamique, sans cesse en devenir.

\subsection{La mise en place des marches urbaines exploratoires de nuit :}

La méthodologie des marches urbaines exploratoires de nuit que nous avons mise en place se base sur ce corpus méthodologique. Il s'agissait de renouveler ces démarches pour effectuer une critique in situ des nuits urbaines. Ce faisant, une culture commune des nuits parisiennes pouvait naître dans les échanges entre les diverses catégories de participants (chercheurs, élus, étudiants, associatifs, techniciens, architectes-urbanistes, exploitants, citoyens, etc.). Nous avons tenté d'articuler cette « objectivation» des nuits urbaines, avec la perception de ces espaces-temps et l'effet produit sur soi des ambiances ${ }^{7}$, en termes physiques, sociaux et psychologiques (sentiments de confort, de sécurité, de propreté, de bien ou mal-être). Ainsi, ce sont à la fois des représentations mentales et formes de perception de l'espace-temps nocturne qui sont interrogées, et les manières dont se déroulent les interactions entre le milieu, ses «prises » et les comportements individuels et collectifs.

Afin d'affiner la méthodologie de diagnostic urbain, nous nous sommes appuyés sur deux types de méthodologies. Les «diagnostics en marchant» sont des dispositifs mis en place par les pouvoirs publics et permettant de faire appel à l'expertise d'usage des habitants d'un lieu afin de permettre un réaménagement adapté. Néanmoins, il s'agit d'une vision fonctionnaliste, basée sur les matrices SWOT (pour forces, faiblesses, opportunités, menaces). De plus, cette évaluation ex ante de définition des axes stratégiques ne permet d'interroger qu'un domaine d'activité et non pas d'obtenir une vision globale. Enfin, elle est souvent mise en

\footnotetext{
${ }^{7}$ L'« ambiance » est une notion du sens commun se révélant être complexe. Elle est composée de trois dimensions : le sensible (ce qui est repérable par les sens de l'humain), le perçu (comment est qualifié ce qui est repéré), le vécu (comment se déroule l'expérience en situation). Ainsi, en parlant des ambiances, nous nous intéressons à la fois à un aspect matériel perceptible par les cinq sens; et aux effets psychologiques en termes d'expérience esthétique et physiologique en termes de confort. M. Miranda (2010) définit l'ambiance comme : «La mise en scène sur un site spécifique d'un stimulus élaboré à travers un ensemble d'éléments - physiques, sensibles, et psychologiques - qui enveloppe les usagers leur conférant un certain confort tout en influençant leur expérience sur le site, c'est-à-dire, influençant : (1) l'interprétation et la représentation qu'ils se font du site et (2) leur comportement sur ce site »
} 
œuvre de manière partielle et peu aboutie. De leur côté, les «promenades sociologiques » de M. Pinçon et M. Pinçon-Charlot (2013) sont une manière de rendre intelligible ce qu'ils appellent le «chaos urbain », c'est-à-dire, les principes d'organisation du monde social. Riches de significations et porteuses d'une dimension souvent oubliée : l'histoire sur le temps long des phénomènes et transformations donnés à voir, elles ne sont cependant pratiquées que par ce couple de chercheur qui nous livrent un portrait de Paris assez général et pouvant sembler assez pauvre.

Pour la réalisation de nos marches urbaines exploratoires de nuit (MUEN), en tant qu'approche sensible et diagnostic urbain, nous nous sommes servis de trois événements : le colloque international "The intricacy of walking and the city. Methods and Experiments"8 (MUEN A), le colloque international «Cohabiter les nuits urbaines. Penser, sentir et narrer la vie nocturne ${ }^{9}$ (MUEN B et C) et les «Rencontres européennes de la vie nocturne \#2 ${ }^{10}$ (MUEN D). Ainsi, nous avons pu améliorer notre méthodologie au fur et à mesure des événements et dépasser le critère de la saisonnalité. L'intérêt de s'appuyer sur ces événements était triple : pouvoir maîtriser le déroulement global des explorations et garder une indépendance d'action et de pensée de par la co-organisation de ces événements ; avoir un vivier de participants divers et volontaires car intéressés par les questions de marchabilité et/ou nocturnalité; avoir un espace de présentation de la démarche et d'explicitation des objectifs, techniques employées, etc. Ce dernier point est important: il ne s'agit pas de laisser les participants seuls face à euxmêmes, mais bien de donner une cohérence au projet, qu'il puisse leur faire sens. Ainsi, nous avions un temps de parole lors de chaque événement et un temps de restitution collective pour les deux derniers événements. Au préalable, chaque participant inscrit devait remplir un questionnaire afin de connaître ses caractéristiques sociodémographiques, son degré de connaissance de la nuit parisienne et son rapport à la nuit urbaine. Cela nous permettait de constituer des équipes en variant les caractéristiques des participants et d'affiner l'analyser en tentant d'amoindrir ce qui serait dû aux caractéristiques individuelles des participants. De même, nous avons pu fournir, en amont, une explicitation de la démarche, sans dévoiler trop de détails afin qu'ils ne se préparent pas en amont et pour éviter de perdre le caractère d'étrangéité de la démarche. Enfin, il nous est apparu essentiel de faire signer un accord de confidentialité afin de garantir le respect de l'éthique du chercheur, des informateurs rencontrés et la préservation du terrain d'enquête.

Divers itinéraires ont été prévus en amont, dans l'objectif de traverser une diversité d'espaces-temps, en ne s'arrêtant pas exclusivement sur les spots festifs nocturnes ${ }^{11}$. Ils ont été pensés en fonction des connaissances de terrain qu'en avaient les organisateurs et, pour la dernière exploration, avec dix-neuf contributions de participants au Conseil de la nuit parisien. Au niveau du tracé, six thématiques principales nous ont permis d'engendrer cette diversité au niveau

\footnotetext{
${ }^{8}$ Labex FutursUrbains, Groupe Thématique « Mobilités Urbaines Pédestres », Paris-Marne la Vallée, 21-23 janvier 2015

${ }^{9}$ Lab’Urba, Prodig, Lavue, Pacte, Paris-Marne la Vallée, 05-06 mars 2015

${ }^{10}$ EuroCouncil of the Night, Pierrots de la nuit, Paris, 17-18 septembre 2015

${ }^{11} \mathrm{cf}$. Annexe ${ }^{\circ} 1$
} 
des formes de : mobilités (infrastructures de transports, potentiels de mobilités et stationnements, etc.), loisirs (commerces de convivialité, loisirs informels, etc.), travail nocturne (services urbains, «ville de garde », etc.), sureté et prévention (sans-abrisme, délinquance, etc.), aménagement urbain (mobilier urbain, voiries, types d'espaces, etc.), information (signalétique, etc.). De même, la volonté était de parcourir un Paris utilisé de nuit, d'amener à la comparaison entre des quartiers aux conditions socio-économiques variées et de traverser des formes de ruptures urbaines, interstices (dont le passage du boulevard périphérique). Il s'agissait donc d'aller à la rencontre d'ambiances et peuples variés, d'espaces-temps vides et animés.

\begin{tabular}{|c|c|c|c|c|}
\hline MUEN & MUEN A & MUEN B & MUEN C & MUEN D \\
\hline Spatialités & $\begin{array}{l}13^{\mathrm{e}} \\
\text { arrondissement } \\
\text { (de la } \\
\text { Bibliothèque } \\
\text { François } \\
\text { Mitterrand à } \\
\text { Denfert- } \\
\text { Rochereau) }\end{array}$ & $\begin{array}{l}\text { a. De la Porte de } \\
\text { la Villette à } \\
\text { la Place de la } \\
\text { République } \\
\text { b. De la Place } \\
\text { Saint-Michel à } \\
\text { la Place de la } \\
\text { République }\end{array}$ & $\begin{array}{l}13^{\mathrm{e}} \\
\text { arrondissement } \\
\text { (de l'avenue } \\
\text { d'Italie } \\
\text { au centre } \\
\text { commercial du } \\
\text { Kremlin Bicêtre) }\end{array}$ & $\begin{array}{l}\text { a. De la Porte de } \\
\text { Pantin } \\
\text { à la Place du } \\
\text { Châtelet } \\
\text { b. De Saint-Denis à } \\
\text { la Place de Clichy } \\
\text { c. De Carreau du } \\
\text { Temple à la Place } \\
\text { du Châtelet }\end{array}$ \\
\hline Temporalités & $\begin{array}{l}23 / 01 / 2015 \\
21 \mathrm{~h} 30-23 \mathrm{~h} 30\end{array}$ & $\begin{array}{l}05 / 03 / 2015 \\
\text { a. } 23 \mathrm{~h} 00-02 \mathrm{~h} 30 \\
\text { b. } 21 \mathrm{~h} 00-00 \mathrm{~h} 30\end{array}$ & $\begin{array}{l}06 / 03 / 2015 \\
23 \mathrm{~h} 00-01 \mathrm{~h} 00\end{array}$ & $\begin{array}{l}\text { 17/09/2015 } \\
21 \mathrm{~h} 30-05 \mathrm{~h} 00\end{array}$ \\
\hline
\end{tabular}

\subsection{Le déroulement des marches urbaines exploratoires de nuit :}

Lors de ces marches urbaines exploratoires de nuit, nous avons tenté d'obtenir un diagnostic urbain critique et une chrono-cartographie sensible de Paris. Chaque membre du groupe consignait les éléments non-humains, humains et ressentis sur les espaces et temporalités nocturnes traversés. Les MUEN A et $\mathrm{C}$ étaient plutôt centrées sur les représentations et perceptions des participants: chacun les notaient sur un carnet et se devait de réaliser une carte mentale à la fin du parcours. Puis, une discussion collective avait lieu sur ces perceptions et représentations de la nuit parisienne traversée, en prenant appui sur leur propre carte mentale, afin de partager ses expériences sensibles. Les MUEN B et D tentaient d'articuler l'objectivation des aménagements urbains et des représentations et perceptions sensibles des nuits urbaines parisiennes. Pour cela, chaque membre du groupe se voyait remettre un carnet d'exploration ${ }^{12}$ contenant divers objectifs à atteindre. Concernant les diagnostics urbains, des points précis étaient indiqués pour effectuer une observation directe, suivant une grille semiouverte préétablie ; ainsi que des tranches d'itinéraires d'environ dix minutes pour effectuer une observation «flottante » et remplir une grille ouverte préétablie. De même, des grilles d'interviews ont été mises en place, l'une pour interroger des noctambules présents et l'autre pour interroger des travailleurs de nuit. Nous pouvons noter que la prise de photographies, partagées par la suite, était conseillée. Concernant la chrono-cartographie sensible, la réalisation d'une carte mentale (accompagnée d'un discours écrit) était demandée en fin de parcours, ainsi qu'une grille d'impressions (au début et à la fin du parcours), sur les

${ }^{12} \mathrm{cf}$. Annexe $\mathrm{n}^{\circ} 2$ 
souvenirs du trajet effectué jusque-là et les sensations corporelles.

La réalisation d'observations directes (H. Peretz, 2004 ; AM Arborio, P. Fournier, 1999 ; etc.), d'observations flottantes (C. Pétonnet, 1982) et d'entretiens informels (P. Bruneteaux, C. Lanzarini, 1998) sont des techniques d'enquête aujourd'hui éprouvées, validées par la communauté scientifique. Nous les avons adaptées au contexte d'une enquête en mouvement, en marche, et pour des enquêtés n'étant en partie pas formés sur le long terme à ces techniques. La réalisation de cartes mentales est, cependant, une méthode moins connue. Il convient de distinguer la « carte mentale » (ou cognitive map) de la «carte heuristique » (ou mind map), qui s'intéresse à la structuration de la pensée. La carte mentale serait la retranscription sur papier d'une cartographie de son expérience sensible de son environnement. Environnement et individu sont articulés et c'est la manière dont l'individu se représente l'environnement (suite à sa perception de cet environnement) qui intéresse le chercheur, par le médium du dessin. K. Lynch est l'auteur de référence à propos du développement de cette méthode (1960). Il a démontré la légitimité de l'analyse visuelle du paysage urbain.

Nous avons suivi la technique de K. Lynch qui consiste à présenter au participant une feuille blanche vierge, de format A4. Par contre, nous n'avons pas demandé de dessiner des trajets connus et habituels, mais, de représenter leur exploration nocturne après coup, en nommant les points remarquables et les ressentis. Tel que le note V. Poublan-Attas (1998), il s'agissait d'un dessin libre du déplacement effectué, en suivant les instructions de l'enquêteur. Cependant, quelques questionnements peuvent se poser à propos de cette technique d'enquête, notamment autour de sa fiabilité. Est-il réellement possible de faire correspondre nos représentations mentales à une représentation visuelle ? Il semble nécessaire de compléter cette technique d'enquête avec d'autres techniques, telle que la réalisation d'entretiens (G. Moser, K. Weiss, 2003). Ces derniers permettent de comprendre finement la manière dont les enquêtés ont raisonné pour construire leur carte mentale et le sens qu'ils lui donnent. Elles ont tout de même le mérite d'exprimer des éléments constitutifs de la représentation mentale qui sont difficiles à exprimer verbalement, lors d'un entretien; des éléments relevant de l'implicite.

\section{Les résultats empiriques et méthodologiques obtenus par les MUEN :}

La mise en place des MUEN, consistant en une forme de «bricolage » méthodologique évolutif en fonction des retours des participants et chercheurs, est un processus itératif. C'est par les analyses successives des résultats empiriques obtenus que nous avons pu réorienter la méthodologie. Elle ne se veut pas figée et se doit d'être adaptée aux contextes culturels.

\section{Les analyses des données obtenues :}

Afin d'établir la chrono-cartographie sensible, l'analyse des cartes mentales a été effectuée à partir des conseils de K. Lynch, en articulant trois éléments : le dessin 
de la carte mentale, la scripturalité de la carte mentale et le discours sur la carte mentale.

Pour le dessin, différentes échelles d'analyse s'imposent: la composition du dessin, la localisation des objets et leurs relations. Tout d'abord, d'un point de vue global, il nous semble intéressant d'analyser: l'échelle globale du dessin par rapport à l'échelle réelle; le type de représentation (heuristique, séquentielle, cartographique, etc.) ; l'orientation du dessin. Puis, nous portons notre intérêt sur des points précis, relatifs à la grille de K. Lynch : les voies (le type de réseau, la hiérarchisation des voies, etc.), les limites (les frontières, ruptures, coutures), les quartiers (les parties de la ville identifiées comme un tout), les nœuds (les points stratégiques de jonction et de concentration, où la prise de décision se fait) et les points de repère (les références ponctuelles en fonction de caractéristiques physiques ou symboliques, l'échelle d'action d'un point de repère). Concernant la scripturalité, nous portons notre intérêt sur les mots et expressions inclus dans le dessin. Nous commençons par classer ces termes en fonction de leur nature (jugement climatique, jugement esthétique, etc.). Puis, nous articulons ces termes avec l'analyse du dessin de la carte mentale. Enfin, nous comparons les scripturalités entre les individus. À propos du discours, nous portons notre intérêt sur le récit effectué après la réalisation de la carte mentale. Il s'agit, tout d'abord, d'évaluer la validité des interprétations précédentes du fait d'une explicitation orale. Puis, nous cherchons à comprendre les convergences et divergences entre les participants, par la structure du discours. A la fin de ces trois parties, nous catégorisons les cartes mentales et tentons de les articuler aux caractéristiques sociodémographiques, à l'investissement de cet espace-temps, à la connaissance de la pratique sociale et au background scolaire des individus. Enfin, nous tentons de retirer des pistes sur les représentations mentales des nuits parisiennes, en fonction de la perception de cet espace-temps. Cela ne donne pas une représentation cartographique, au final, où il serait vain d'exprimer les ruptures, détournements et, surtout, d'exprimer les variations en fonction des temporalités et des activités sociales dynamiques.

Afin d'établir le diagnostic urbain, nous avons effectué une analyse thématique, proche des conseils de J. Poirier pour les récits de vie (1996) ${ }^{13}$. L'analyse catégorielle était composée de cinq dimensions : les sens des ambiances; les aménagements et dérangements urbains; l'(in-)hospitalité nocturne; les (im)mobilités et le (dés-)ordre urbain. Celles-ci ont été mises en lumière via les quatre axes d'analyse d'ensemble :

- la conception et l'aménagement des espaces-temps urbains (configuration, mobilier, etc.) ;

- la gestion de l'espace urbain (propreté, entretien, maintenance, sécurité, etc.) ;

- les usages (signes visibles d'appropriation, de dysfonctionnement, de conflits ou de dégradation, etc.) ;

\footnotetext{
${ }^{13}$ Il s'agit d'une analyse de contenu dont nous avons retenu une partie des étapes : relecture des fiches et carnets, avec annotations ; clarification du corpus et mise en cohérence ; organisation du corpus via des grilles d'analyse ; traitement catégoriel et quantitatif ; synthèse ; interprétations et commentaires
} 
- les discours sur ces espaces-temps, pour leur promotion et la manière dont ils sont perçus.

Chaque dimension a été analysée par ces quatre axes pour interroger une dialectique. Elles peuvent être regroupées en un schéma :

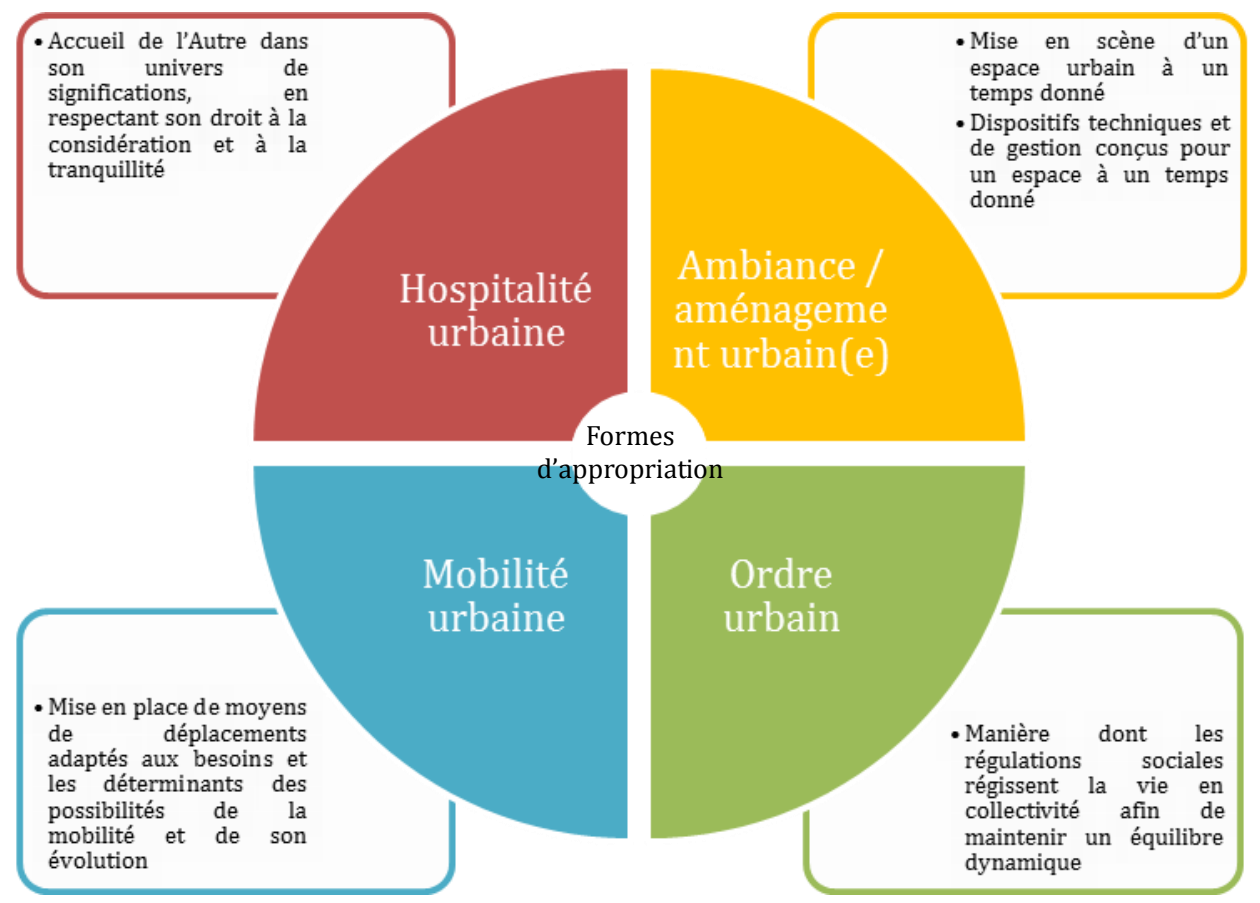

Ces cinq dimensions sont des éléments centraux pour comprendre comment est occupé un espace-temps urbain nocturne, les formes de "publicisation » et de perception/représentation.

\section{Les détours méthodologiques :}

Les résultats donnés ci-dessus, de manière rapide, donnent l'impression d'une grande homogénéité. Ce serait omettre les caractéristiques individuelles, où les critères de nationalité et socioprofessionnels jouent un rôle important dans les différenciations de représentations sociales et perceptions individuelles. De même, la qualité des données n'est pas homogène, elle a évolué en fonction des variables testées et des améliorations successives apportées à la méthodologie des MUEN.

$\mathrm{Au}$ niveau méthodologique, nous avons souhaité tester trois variables: temporalités (horaires, saisons, jours), spatialités, constitution et fonctionnement des collectifs. Par rapport aux temporalités, bien que la plupart des parcours durent trois heures (sauf pour la dernière exploration où ils duraient plutôt 05-06 heures), une temporalité large de la nuit a été traversée : de $21 \mathrm{~h} 30$ à $05 \mathrm{~h} 00$, avec une forte présence au moment de la temporalité de rupture nocturne $(00 \mathrm{~h}-02 \mathrm{~h})$. Les explorations ont toutes eu lieu un jeudi ou un vendredi, soit des jours particulièrement marqués par les activités nocturnes (dont festives). En ne choisissant pas le samedi soir, nous pouvions observer le chassé-croisé dans la rue entre les derniers noctambules et premiers travailleurs. De plus, le jeudi soir, spécifiquement « réservé » aux soirées étudiantes, semble en perte de vitesse en 
termes de chiffre d'affaire et de fréquentation pour les établissements de loisir nocturne, contrairement au vendredi soir. Au niveau des spatialités, nous avons pu voir la diversité des parcours traversés. Nous pouvons noter également que la première marche était la seule où il n'y avait aucun parcours prédéfini : seuls les points de départ et d'arrivée avaient été donnés, contrairement aux autres. Enfin, concernant le fonctionnement et la constitution des collectifs, la MUEN A était constituée de cinq groupes de 3-5 personnes, où des individus de nationalité diverse étaient mixés avec un individu Parisien (aussi pour des raisons pratiques), afin de relever les différences d'objectivation et de perception suivant la culture d'origine. Cependant, l'individu Parisien a bien souvent «embarqué » les autres membres dans une direction, voire avec une allure pouvant empêcher l'observation. La MUEN B était formée de deux groupes de 5-6 individus chacun, dont l'un était composé plutôt d'individus ne connaissant pas bien la vie nocturne de Paris et l'autre composé plutôt d'individus en ayant une connaissance préalable. Les organisateurs guidaient alors les groupes. La MUEN C s'est déroulée avec 8 individus, menés par deux artistes du collectif $\mathrm{KMK}^{14}$. L'originalité, ici, était l'interdiction d'échanger des paroles entre les participants, afin de se laisser personnellement guider et de favoriser l'attention au milieu urbain. La MUEN D était constituée de deux groupes de 7 individus (en diversifiant les catégories d'acteurs) et d'un groupe de 14 individus (en diversifiant les catégories d'acteurs et nationalités), menés de manière souple par les organisateurs.

Voici un tableau permettant de récapituler les différenciations testées :

\begin{tabular}{|c|c|c|c|c|c|}
\hline & & MUEN A & MUEN B & MUEN C & MUEN D \\
\hline \multirow{2}{*}{ Jour } & Jeudi & & + & & + \\
\hline & Vendredi & + & & + & \\
\hline \multirow{3}{*}{ Horaire } & $21 h-00 h$ & + & + & + & + \\
\hline & $00 h-02 h$ & & + & + & + \\
\hline & $02 h-05 h$ & & & & + \\
\hline \multirow{2}{*}{ Climat } & Plutôt froid & + & + & + & \\
\hline & Plutôt chaud & & & & + \\
\hline \multirow{2}{*}{ Parcours } & Tracé & & + & + & + \\
\hline & Libre & + & & & \\
\hline \multirow{4}{*}{ Quartiers } & Sud-Est & + & & + & \\
\hline & Nord-Est & & + & & + \\
\hline & Centre & & + & & + \\
\hline & Nord-Ouest & & & & + \\
\hline \multirow{3}{*}{$\begin{array}{c}\text { Constitution des } \\
\text { groupes }\end{array}$} & $3-5$ & + & & & \\
\hline & $5-8$ & & + & & + \\
\hline & $+d e 8$ & & & + & + \\
\hline \multirow{4}{*}{$\begin{array}{c}\text { Fonctionnement } \\
\text { des groupes } \\
\text { (homogénéité } \\
\text { sexuelle, socio- } \\
\text { professionnelle, en } \\
\text { âges, de degrés de } \\
\text { connaissance, }\end{array}$} & $\begin{array}{c}\text { Nationalités } \\
\text { diverses }\end{array}$ & + & + & & + \\
\hline & $\begin{array}{c}\text { Nationalités } \\
\text { homogènes }\end{array}$ & & + & + & + \\
\hline & Echanges verbaux & + & + & & + \\
\hline & $\begin{array}{c}\text { Interdiction des } \\
\text { échanges }\end{array}$ & & & + & \\
\hline
\end{tabular}

${ }^{14}$ La compagnie artistique KMK, a produit une collection de promenades intitulée « Ailleurs à... », sur divers territoires et pour obtenir une cartographie sensible des espaces traversés. La marche est un "processus d'exploration » avant de devenir un moyen de restitution. Les perceptions peuvent être modifiées par un accompagnement sonore 


\begin{tabular}{|c|c|c|c|c|c|}
\hline respectée) & & & & & \\
\hline \multirow{2}{*}{ Type de MUEN } & Sensible & + & + & + & + \\
\cline { 2 - 6 } & Diagnostic urbain & & + & & + \\
\hline
\end{tabular}

La différenciation en termes horaires est articulée au climat, c'est-à-dire, à la difficulté de continuer la marche dans le froid et au degré d'animation nocturne. Le parcours «libre» rendait l'analyse complexe et faible du fait du peu de représentativité, cependant, il permet de montrer des attractions sensorielles fortes de par la proximité des cheminements, les détours étant dus aux connaisseurs d'un lieu particulier. Néanmoins, de son côté, le «tracé » ne suit pas les trajectoires festives ou des noctambules mais oblige à traverser les formes de diversité notées dans les oppositions analytiques, qui n'auraient sans doute pas été explorées. Les quartiers Ouest et Sud ont peu été traversés du fait de la moindre animation nocturne, mais aussi de la réduction du nombre de parcours via un manque de participants. Les plus petits groupes se sont avérés plus efficaces, étant donné qu'au-dessus de huit participants il y avait une division en sous-groupes, et assuraient une plus grande cohésion évitant les abandons. Le mélange de nationalités et des autres caractéristiques encourageait à des échanges plus fructueux que des groupes homogènes. Le fait d'interdire l'échange semble, par contre, plus efficace pour éprouver la ville de manière sensible, se laisser porter, mais, est difficilement accepté et compris au début. Enfin, le diagnostic urbain pouvait très bien se mêler à la radiographie sensible, cependant, la sélection fine d'une thématique aurait permis de l'approfondir (tout en perdant la vision globale qu'il aurait fallu reconstruire après-coup, en multipliant les parcours).

Par conséquent, la variable temporelle se doit d'être adaptée aux espaces traversés en termes d'animation urbaine et au climat. La variable spatiale est davantage efficace quand l'espace est circonscrit, au moins en partie, quand le parcours est guidé de manière souple et les espaces traversés sont différenciés socioéconomiquement et culturellement. La variable collective doit être maitrisée en termes de nombre, pour assurer une cohésion, et en termes d'échanges, pour permettre une appréhension sensible. Enfin, le type de MUEN se doit d'être bien défini à l'avance, tout en sachant que des pertes de données auront lieu en fonction de ce choix.

\section{Les enjeux heuristiques des MUEN dans le cadre d'une critique socio-urbaine :}

\subsection{Les limites et opportunités des MUEN :}

La mise en place de la méthodologie des marches urbaines exploratoires de nuit s'est effectuée via une prise de distance critique avec les méthodologies de radiographie sensible de la ville et de diagnostic urbain. Ainsi, nous avons construit la méthodologie de manière scientifique, en utilisant des techniques éprouvées par la communauté scientifique et mettant en place une démarche rationnelle d'accès au réel : analyse préalable du territoire vécu, mise en place et test des parcours, réalisation des explorations, analyse des données et interprétations, permettant de revenir sur l'analyse préalable et d'améliorer la 
méthodologie (dans le choix des variables). Les catégories d'analyse ont été construites de manière itérative, en articulant les recherches effectuées préalablement par les organisateurs des MUEN et avec la volonté d'une compréhension complexe du monde urbain nocturne. Le prisme des représentations collectives et perceptions sensibles a été articulé au prisme de l'objectivation des aménagements urbains, soit le respect de la relation dialogique entre recherche de l'objectivité et de la subjectivité, la seule à même de permettre l'obtention d'un discours scientifique sur l'hospitalité urbaine, les formes de publicisation, et d'éviter un regard fonctionnaliste et clos, ainsi qu'un regard individualiste et fractionné. Ce faisant, nous avons tenté de neutraliser les biais des caractéristiques individuelles des participants lors de l'analyse. Chaque participant a pu exprimer son vécu et interprétation des espaces-temps traversés (via les cartes mentales) et énoncer les souvenirs et ressentis provoqués (via les grilles d'impressions et récits s'appuyant sur les cartes mentales). La collection des mini-diagnostics urbains (via les grilles d'observation) a permis d'en faire ressortir une analyse globale, c'est-à-dire, de comprendre les configurations espace-temps-action en situation urbaine, ainsi que d'avoir accès à quelques univers de significations des individus rencontrés en chemin (via les entretiens informels). Chacun a pu effectuer une description, en utilisant ses propres moyens ; description aidée par le carnet d'exploration fournit. Le fait de mélanger les équipes, dont au niveau des nationalités, a permis de s'écarter des «clichés » portant sur un lieu, pour n'en relever que la substance. L'analyse globale a permis de relever des points d'accroche en termes d'interactions entre les conduites sociales, le contexte ou milieu urbain et les «prises » offertes par ce milieu spatiotemporel, donc, de montrer à la fois les ruptures du tissu urbain en termes d'ambiance et d'aménagement et les effets de ces ruptures. Une forme de «culture commune»des nuits parisiennes a été rendue possible, surtout via les petits groupes de collectifs, en permettant le dialogue entre divers types d'acteur, lors des explorations et après-coup (via les restitutions collectives et rapports écrits). Ce dialogue collectif émerge par les événements rencontrés en situation. En effet, le partage de l'expérience longue et épuisante de la marche permet d'amoindrir les statuts sociaux, encourage chacun à s'exprimer, en faisant appel à sa propre expérience d'usager qui sera montée en généralité par le dialogue. La différenciation des âges de la vie et de l'introduction dans les nuits parisiennes encourage également à avoir un retour sur la dimension historique. Néanmoins, la phase de "préconisations » n'a pas été entamée, du fait qu'il semble prématuré de l'effectuer avant et pendant l'analyse des données. Il est essentiel de les relier aux problématiques rencontrées et ne relèvent pas du métier d'un chercheur, plutôt des techniciens des pouvoirs publics. Le chercheur n'est qu'une aide à la décision publique, pointant les processus en cours et passés, ainsi que leurs conséquences, ici, socio-urbaines.

Cependant, la méthodologie des MUEN n'est pas parfaite, un certain nombre de limites se sont posées. Tout d'abord, nous n'avons pas réellement eu accès aux micro-rituels du quotidien, étant donné que la part d'étrangéité à l'espace-temps était une volonté initiale de la démarche. Il s'agissait d'éviter de rentrer dans le territoire « intime» des participants, afin d'avoir la confrontation avec un regard 
critique, avec des yeux d'un Persan (Montesquieu, $1721^{15}$ ) étant en partie familier de la culture occidentale. Les contrastes culturels permettaient alors de mieux appréhender le territoire, nous étant familier en tant que terrain d'enquête. D'ailleurs, la collection de regards collectifs est un moyen d'enrichir les observations du chercheur, notamment autour de la validité de ses interprétations au regard d'un prisme qui aurait pu devenir socio-centrique. De même, nous n'avons pas eu accès directement aux trajectoires festives ou de noctambules, du fait d'un tracé spatio-temporel menant à la confrontation de la diversité et donc à l'ouverture des perspectives face aux connaissances d'usager. Celles-ci n'ont pu être obtenues qu'en partie via les entretiens informels. Néanmoins, cette interrogation n'était pas un objectif affiché à la démarche. Cependant, une des limites fortes est le manque de formation des participants-enquêteurs qui, quel que soit leur statut social (et même concernant les chercheurs), sont peu habitués à ce type de démarche qui ne leur fait pas complètement sens. De la sorte, les descriptions et autres recueils de données ont été bien souvent parcellaires et approximatifs. La présence des chercheurs participant à l'exercice a permis de palier à ce handicap. Ceci peut également s'expliquer par les outils proposés qui demandaient une attention soutenue et un temps de réponse important, afin de garantir la richesse des données. Un équilibre est à tenir entre la nécessité de mettre en exergue les informations nécessaires à recueillir (afin d'éviter des oublis tels que les caractéristiques sociodémographiques et de pouvoir comparer les collections de diagnostics pour les analyser) et la liberté d'action des participants, dans des interactions informelles et éphémères. Notons que les grilles d'observation ont tendance à figer la vision d'un lieu à un instant $t$. Elles sont davantage adaptées à la description des aménagements urbains qu'à la description fine des usages et usagers. Seule la répétition des mêmes parcours permettra d'y avoir accès, au moins partiellement. Nous avons dû faire face aussi à la difficulté d'accès à des participants venant de la société civile, et sous ce statut, malgré la publicité des événements effectuée sur Internet et dans des magazines parisiens. Ceci a pu limiter, parfois, les échanges entre acteurs différenciés et amener à une participation plutôt d'individus concernés directement par les problématiques nocturnes. A contrario, la mise en place d'une telle plateforme d'échanges peut amener à une richesse non maîtrisable d'échanges s'éloignant du cœur des objectifs initiaux (notamment concernant les descriptions de cartes mentales, où les participants échangeaient davantage sur l'expérimentation et autres sujets de digression que sur la radiographie sensible de la ville). Enfin, et tel que l'avait pressenti GE Debord, la réalisation des marches urbaines exploratoires est plus difficile la nuit qu'en journée. En effet, au cœur de la nuit, les sollicitations urbaines se font plus rares. De même, le climat se rafraîchit et la fatigue se fait ressentir de par le creux physiologique. Ainsi, un certain nombre d'abandons a pu être noté en cours de route pour les plus longues marches nocturnes. Les abandons préalables à la marche sont plutôt dus au fait que des participants voient que ces MUEN ne convergent pas forcément avec leurs intérêts singuliers, ce qui est dommageable. Donc, une communication efficace et pertinente semble importante pour le bon déroulement de la méthodologie.

\footnotetext{
${ }^{15}$ Dans ses Lettres persanes, Montesquieu donne une illustration aboutie du « regard étranger », c'est-à-dire, d'un regard interrogateur sur des mœurs étrangères qui permet, in fine, d'apporter une critique à ses propres mœurs
} 


\subsection{Les enjeux de la marche dans le cadre des MUEN :}

La spécificité de la méthodologie présentée ici vient du fait qu'elle soit liée à la marche urbaine, donc, à l'ensemble de ses problématiques. La marche peut être comprise, de manière analytique, en mettant en interaction quatre dimensions : technique, logique, sociologique et axiologique.

En effet, avec M. Mauss, nous pouvons considérer la marche comme un ensemble de gestes corporels techniques acquis par l'apprentissage (via l'éducation ou l'imitation) relatif à son environnement social. Il définit les techniques comme «les façons dont les hommes, société par société, d'une façon traditionnelle, savent se servir de leur corps »(M. Mauss, 1950 : 366). Ainsi, la démarche a une efficacité biologique car elle produit des émotions chez celui qui la perçoit (il prend l'exemple de la parade sexuelle), mais elle permet aussi d'accéder aux conceptions de la personne et de sa position dans les mondes social et naturel. Cette démarche est utile, expressive et instituée socialement, c'est-à-dire, relative à un univers de significations partagées. Pour analyser cette dimension en profondeur, nous aurions eu besoin de filmer les participants lors des MUEN. Cependant, nous pouvons noter une démarche différenciée suivant les nationalités présentes avec, par exemple, un pas plus lent et un ensemble de pauses discursives pour les individus américano-latino. De même, lors des MUEN en période froide, la marche se voulait plus rapide et le corps plus renfermé sur soi afin de faire signe d'une nécessité de chaleur, de la recréation d'un cocon. Globalement, la démarche se voulait, au niveau du rythme, relative à une déambulation occidentale, au rythme de la promenade, sous la forme de piétinements facilitant les détours du corps et du regard pour se livrer aux diverses sollicitations. Quand le rythme fut accéléré, il a été noté un manque de temps pour appréhender le milieu urbain et ses ambiances, un resserrement du groupe autour d'un objectif commun de déplacement vers un certain point, ainsi que des regards vers l'autre et ses discussions. Le fait d'être en collectif encourageait à homogénéiser le rythme, les digressions de rythme et de trajectoire étant davantage notables en cas d'absence d'échanges verbaux. Nous pouvons penser que l'échange verbal nécessite l'orientation du corps vers celui auquel on s'adresse, au moins de la tête, et une distance suffisamment proche pour être entendu et ne pas troubler les autres interactions possibles sur place. Néanmoins, au fil de la marche et de la fraîcheur du climat, le rythme ralentissait et des sous-groupes de marcheurs avaient tendance à se créer suite aux accointances. Pourtant, certains s'étaient préparés à vivre cette expérience sous le mode « randonnée », avec des chaussures adaptées, un bonnet, une doudoune, un sac à dos, etc., soit tout un équipement pour parer à ces éventualités, non efficace en soi tant l'habitude de la marche et l'éducation à la patience jouaient des rôles importants.

Mais, le fait de marcher exprime aussi un certain «être-au-monde », un rapport à l'autre et au soi, exprimé sous la forme d'un rôle social, que nous exprimerons avec la perspective d'E. C. Hughes (1996). Ce rôle social pourrait se lire à plusieurs niveaux. Le rôle social du participant se défini comme étant le rôle qu'il croît devoir jouer et le rôle qui lui est assigné, ce qui est attendu de lui. Ainsi, le 
rôle attendu, par les chercheurs, est que le participant soit un enquêteurobservateur en marche, attentif tel que s'il menait une enquête policière. Cependant, cette attente pouvait rentrer en contradiction avec le rôle que les participants croyaient devoir jouer: être un chercheur expérimentant la méthodologie pour la critiquer, être un acteur de la vie nocturne guidant les autres par son savoir d'expert, être un élu politique valorisant sa ville au niveau de l'action publique, etc. De même, le rôle attendu par le chercheur pouvait être redéfini par les autres participants, ce qui pouvait mener, par exemple, à une égalisation de statut social (en considérant que l'autre n'est pas un citoyen ou élu mais un observateur au même titre que moi) ou à une digression de la méthodologie (en considérant que l'autre, en tant qu'expert, aura un discours plus pertinent que moi). Donc, ce rôle social attendu renvoie à un ensemble de règles fixées à l'origine par les organisateurs, auxquelles les participants doivent se conformer. Cependant, elles sont appropriées pour déterminer qui est admis ou exclu du groupe professionnel. De la sorte, celui qui ne rentrait pas dans la définition partagée collectivement se voyait mis à l'écart et peu valorisé. Cela est un moyen de renforcer la solidarité du groupe qui, par l'expérimentation demandée, pouvait sentir être perçu comme étrange aux yeux des autres noctambules présents. Le fait de donner un nom à ce rôle social, de considérer les participants comme des «enquêteurs » participant à une expérimentation scientifiquement contrôlée et dans un cadre institutionnel, permettait de satisfaire en partie à la quête de reconnaissance face au public présent.

Egalement, la marche est «axiologique », elle renvoie à un certain nombre de normes marquées par la maîtrise du désir, l'éthique, le vouloir. Sur la scène sociale de la vie urbaine, les participants ont pu définir leur tâches techniques à effectuer comme étant plus ou moins ingrates et, donc, exprimer leur volonté ou non de les effectuer. Ceci a pu notamment s'exprimer via le rendu de feuilles parfois vierges, montrant un désintérêt à l'expérimentation, au profit de l'intérêt des échanges collectifs et de la découverte d'un autre Paris. De même, des gestes d'énervement et de lassitude se laissaient entrevoir, sans être forcément exprimés verbalement par respect pour les guides. Pour les moins désireux d'effectuer cette expérimentation, l'abandon fut assez rapide, voire avant le commencement. Mais, par respect pour leur engagement et par éthique de professionnel (chercheur, acteur professionnel de la vie nocturne, etc.), nombre de participants ont été jusqu'au bout de l'expérimentation. C'est un véritable travail sur soi, un contrôle de ses désirs immédiats qui est nécessaire mais douloureux pour accoucher des MUEN, c'est-à-dire, se prouver la capacité d'un dépassement de soi et permettre la réalisation d'une entreprise collective. D'ailleurs, nous pouvions noter un certain relâchement du «sang-froid » défini par M. Mauss comme : « un mécanisme de retardement, d'inhibition de mouvements désordonnés ; ce retardement permet une réponse ensuite coordonnée de mouvements coordonnés partants alors dans la direction du but alors choisi. Cette résistance à l'émoi envahissant est quelque chose de fondamental dans la vie sociale et mentale » (Ibid.). Le contrôle du corps, au niveau de sa droiture et de la présentation d'une façade mettant à son avantage, avait tendance à s'amoindrir au profit de l'apparition de mouvements désordonnés, d'un lâcher-prise relatif à l'émoi de par une déstructuration progressive de sa façade présentable (cheveux décoiffés, odeurs de transpiration, 
joues rouges et rhume, etc.), comme forme de résistance au désir de s'en échapper pour vivre une nuit festive ou confortable.

Enfin, l'acte de marcher est «logique », il amène à une capacité de signe, au langage et ce même lorsque les échanges verbaux étaient interdits. En effet, les regards et gestes échangés sont aussi parlant et significatifs que le verbe, mais et surtout, la réalisation de la MUEN amenait à un discours de partage d'expérience, après-coup, de nécessité de verbaliser son vécu. Ceci peut expliquer l'efficacité de la mise en co-présence d'acteurs différenciés, sous un rôle social commun, en suivant la même technique et partageant les mêmes désirs, pour mener au dialogue avec l'autre, s'ouvrir à son univers de signification et tenter de le comprendre. De la sorte, le langage portait sur, dans et par la marche, sur la fatigue et la douleur, le rythme et la manière de l'effectuer, lors de la marche et des sollicitations urbaines, dans un partage de son regard sur ces sollicitations (tels deux verres de vin pleins trouvés par terre devant des grilles, où chaque participant donnait son interprétation, son possible du phénomène passé), et grâce à la marche en tant qu'expérimentation partagée, créatrice d'une cohésion sociale.

Par conséquent, au-delà des objectifs initiaux fixés par la méthodologie des MUEN, nous avons pu en découler une interprétation de la marche urbaine, en situation. Ceci nous a permis de comprendre comment la définition d'un rôle social sur la scène du théâtre urbain nocturne, amenait à l'acceptation ou non d'effectuer un certain nombre de tâches, tâches permises par une technique du corps signifiant son milieu social et permettant la création d'une cohésion sociale, d'un univers collectif de significations.

\subsection{De la marche à la critique socio-urbaine :}

Nous avons pu voir que la marche, dans le cadre des MUEN, est une méthodologie d'analyse urbaine et sensible, mais, c'est aussi un moyen pour permettre la participation citoyenne et un déplacement spécifique, avec toutes ses conséquences. Ce faisant, nous avons pu procéder à une critique socio-urbaine, en situation, c'est-à-dire, relative à une configuration espace-temps-action. Cependant, nous avons pu remarquer que les MUEN ne suffisent pas, en soi, au diagnostic critique. Elles sont à inclure dans une démarche plus générale de connaissance préalable du territoire, de ses problématiques et de la construction de catégories analytiques. En fait, elles permettront davantage d'enrichir et de vérifier les interprétations initiales, ainsi que de les porter à plus grande échelle. Il s'agit donc d'une véritable généralisation de recherches effectuées en configuration restreinte, à un niveau micro-, tout en prenant compte des spécificités des autres configurations. En retour, les découvertes spécifiques aux autres configurations et les similitudes avec la configuration initialement étudiée permettront d'enrichir ces dernières. Les MUEN permettent d'effectuer une articulation dynamique entre une situation socio-urbaine micro- et ses impacts macro- à l'échelle sociétale.

Néanmoins, cette critique socio-urbaine des MUEN ne permet pas, en soi, d'analyser les continuités/discontinuités historiques d'avec la société moderne, en 
termes politiques, technico-économiques et identitaires. Or, les auteurs critiques de la société moderne ${ }^{16}$ analysent les ruptures dans l'organisation des rapports sociaux entre la modernité «classique» occidentale et le temps présent. Pour autant, les MUEN permettent d'avoir une emprise empirique face aux essais critiques, parfois spéculatifs. Elles encouragent à la réalisation d'enquêtes de terrain pour renforcer ces critiques de manière concrète, sur sa propre société. En effet, E. C. Hughes note que la connaissance de soi (de sa société) ne peut s'effectuer que si l'on est disposé à connaître l'autre via un nouvel éclairage. Il ne s'agit pas d'une entreprise aisée, mais, l'observateur doit être capable de s'analyser dans son propre rôle social en fonction de la matrice sociale auquel il prend part. Ainsi, si la sociologie peut se définir comme la science spécifiquement portée sur l'analyse des interactions sociales et de leurs impacts culturels et institutionnels, les mêmes problématiques que la vie en société se posent. Donc, le problème d'effectuer une «sociologie de la sociologie » est de reconnaître les situations permettant cela. Oser se confronter directement au regard d'autres, pour un chercheur qui met en place des MUEN, est une épreuve, si elle est acceptée, pouvant mener à un enrichissement personnel et professionnel sans commune mesure.

\section{Bibliographie}

Amphoux, P. (2013), «Les approches sensibles du territoire », DEA Ambiances architecturales et urbaines

Arborio, AM, Fournier, P. (1999), L'enquête et ses méthodes : l'observation directe, Paris : Nathan université

Arendt, H. (1983), Conditions de l'homme moderne, Paris : Calmann-Lévy, $2^{\mathrm{e}}$ éd. ( $1^{\text {ère }}$ éd. en 1958, The Human Condition)

Bruneteaux, P., Lanzarini, C. (1998), «Les entretiens informels », Sociétés contemporaines, vol. $30, \mathrm{n}^{\circ} 1$, pp. 157-180

Cauquelin, A. (1977), La ville la nuit, Paris : PUF

Debord, GE (1958), «Théorie de la dérive », Internationale situationniste, bulletin $\mathrm{n}^{\circ} 2$, Paris : Les sections de l'Internationale situationniste [en ligne]

Galinier, J., Monod Becquelin, A. (2015), « La nuit se lève », Colloque du 18 décembre

Gieseking, JJ (2013), "Where we go from here: the mental sketch mapping method and its analytic components", Qualitive Inquiry, 19: 712

\footnotetext{
${ }^{16}$ Il ne s'agit pas de critiquer l'« époque moderne » au sens historique du terme qui s'étendrait de la fin de l'Empire romain à la Révolution française, voire à la fin de l'Empire napoléonien, mais, bien d'une critique de la société qui leur est contemporaine à partir d'une vision généalogique ou d'une historiographie diachronique du phénomène social étudié
} 
Grosjean, M., Thibaud, JP (2001), L'espace urbain en méthodes, Marseille : éd. Parenthèses, ch. 4-5

Gwiazdzinski, L. (2005), La nuit, dernière frontière de la ville, Paris : Aube

Gwiazdzinski, L. (2006), «Traversées nocturnes », Maud LE FLOC'H, Mission repérage. Un élu un artiste, Montpellier : éd. L’Entretemps, pp. 241-242

Heurgon, E. (2005), «Préserver la nuit pour réinventer le jour, essai de prospective nyctalogique », C. Espinasse, L. Gwiazdzinski, E. Heurgon, La nuit en question(s), Paris : éd. de l'Aube

Hughes, E. C. (1996), Le regard sociologique, Paris : Ecole des Hautes Etudes en Sciences Sociales, textes rassemblés et présentés par JM Chapoulie

Lévi-Strauss, C. (1960), La Pensée sauvage, Paris : éd. Plon, p. 27

Lynch, K. (1969), L’image de la cité, Paris : Dunod (trad. Marie-Françoise Vénard et Jean-Louis Vénard)

Mauss, M. (1950), «Les techniques du corps », Sociologie et anthropologie. Paris : PUF, pp. 366-386 ( $1^{\text {ère }}$ éd. dans Journal de psychologie, 1936, vol. 22, n $\left.{ }^{\text {os}} 3-4\right)$

Miranda, M. (2010), «L'événement urbain festif : vers une «gestion de site exploratoire » sur l'espace public ? Les cas de Nantes et de Bordeaux », thèse de doctorat en Géographie, dir. A. Bourdin, Université Paris-Est, 22 novembre

Peretz, H. (2004), Les méthodes en sociologie : l'observation, Paris : La Découverte

Pétonnet, C. (1982), «L'observation flottante. L'exemple d'un cimetière parisien », L'Homme, vol. 22, n 4, pp. 37-47

Pincon, M., Pincon-Charlot, M. (2013), Paris. Quinze promenades sociologiques, Paris : Payot et Rivages, ( $1^{\mathrm{re}}$ éd. Calmann-Lévy, Paris Mosaïque. Promenades urbaines, 2001)

Piveteau, V., Lardon, S., (2005), « Méthodologie de diagnostic pour le projet de territoire une approche par les modèles spatiaux », Géocarrefour, vol. 80, n², pp. $75-90$

Platon (2015), De la République. Livre VII. L'allégorie de la caverne, trad. de B. Suzanne [en ligne]

Poirier, J., Clapier-Valladon, S., Raybaut, P. (1996), Les récits de vie. Théorie et pratique, Paris : PUF ( $1{ }^{\text {ère }}$ éd. 1983)

Poublan-Attas, V. (1998), «L'espace urbain déformé : transports collectifs et 
cartes mentales », thèse de doctorat, spécialité Transport, dir. JM Offner, Ecole Nationale des Ponts et Chaussées, LATTS

\section{Annexes}

\section{Cartes des parcours :}

L'ensemble des parcours - testés par nous-mêmes au préalable - est lisible sur les cartes suivantes :

Figure 1: EUNW A, Paris (75013), 01/23/2015, authors

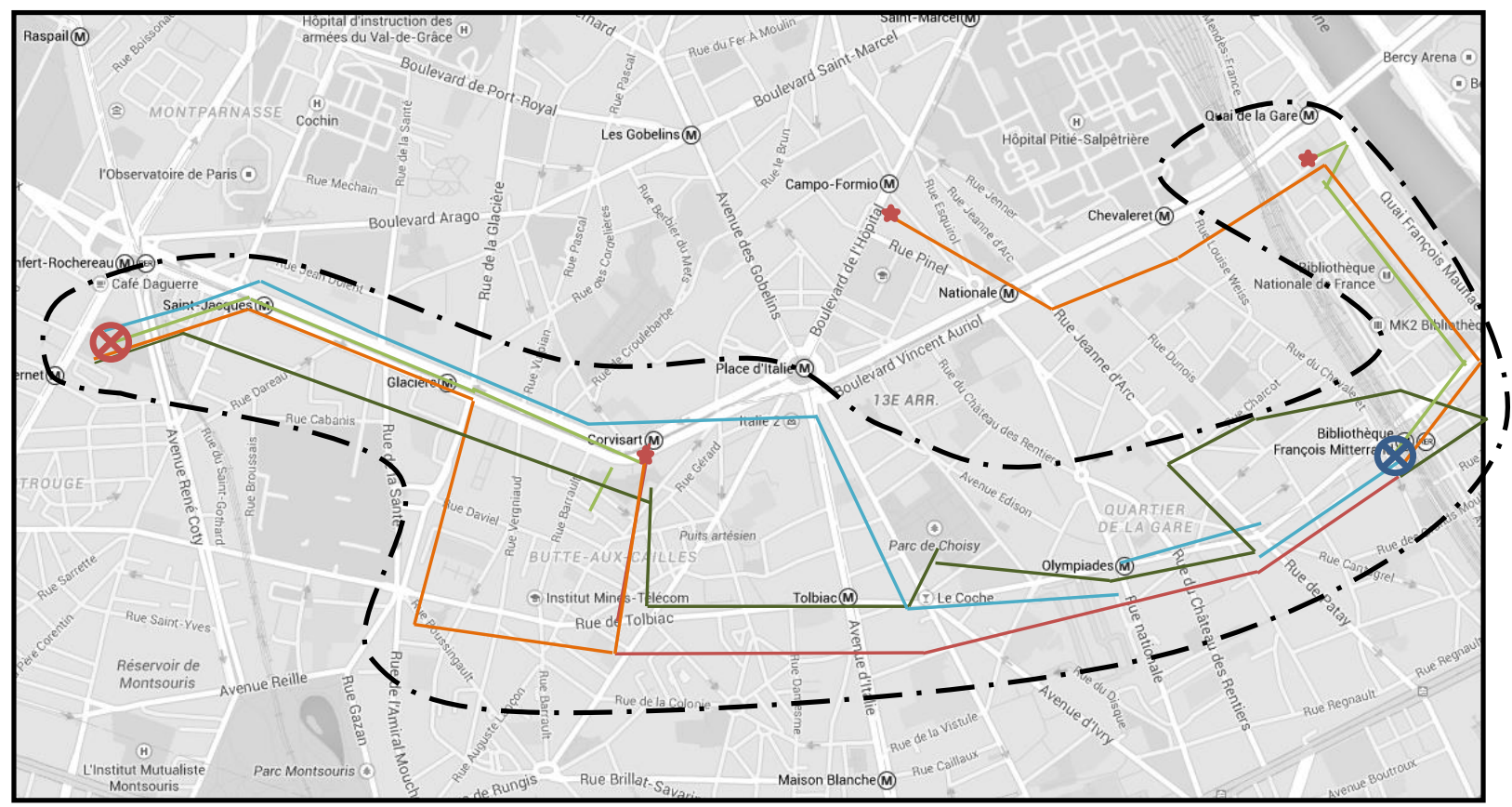

Key:

: Group A

: Group B

_: Group D

: Group E

: Group F

Q : Starting point, Bibliothèque François Mitterrand, 09.30 pm

: Finishing point, Denfert-Rochereau, $11.30 \mathrm{pm}$ 
Figure 2: EUNW B, Paris, 03/05/2015, authors
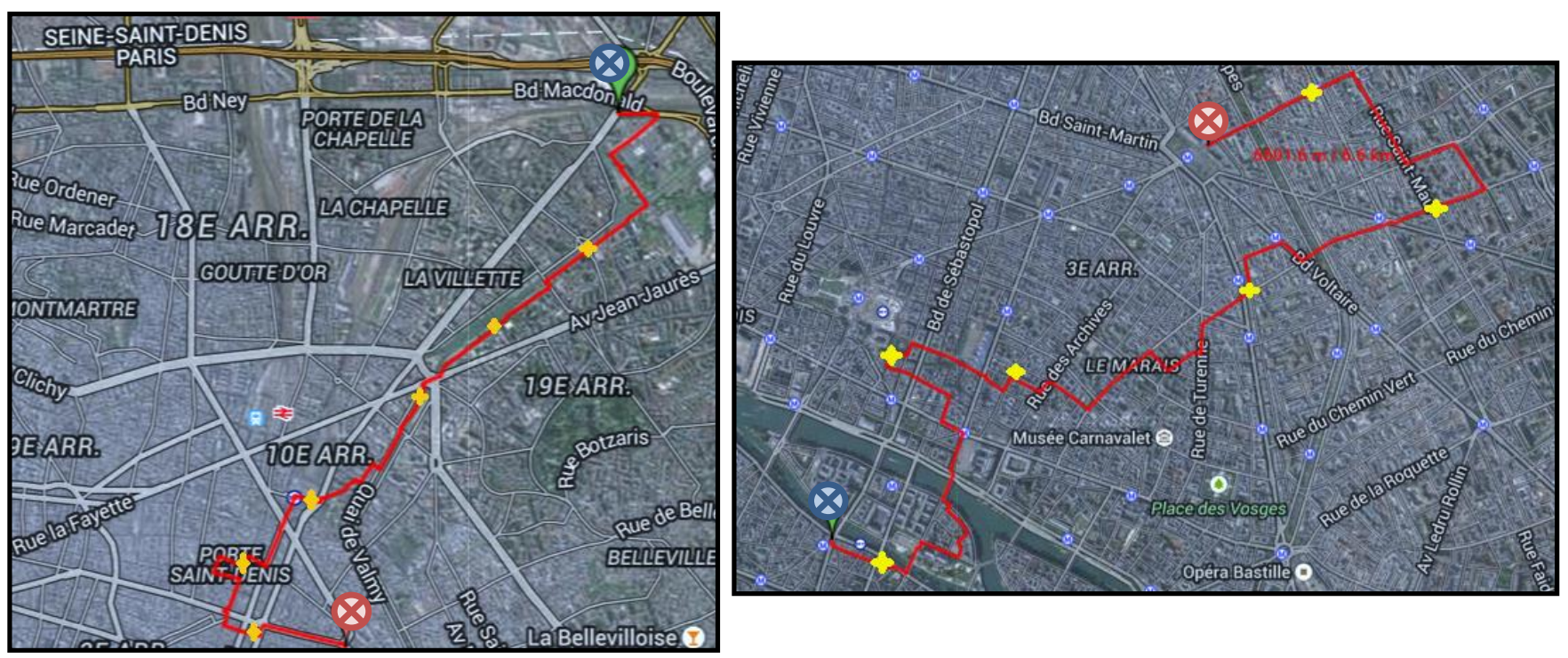

Key:

Q : Starting point, Porte de la Villette, $11.00 \mathrm{pm}$ $09.00 \mathrm{pm}$

8. Finishing point, Place de la République, $02.30 \mathrm{am}$ République, 00.30 am

Key:

: Starting point, Place Saint-Michel,

Q : Finishing point, Place de la

Figure 3: EUNW C, KMK, Paris, 03/06/2015, authors

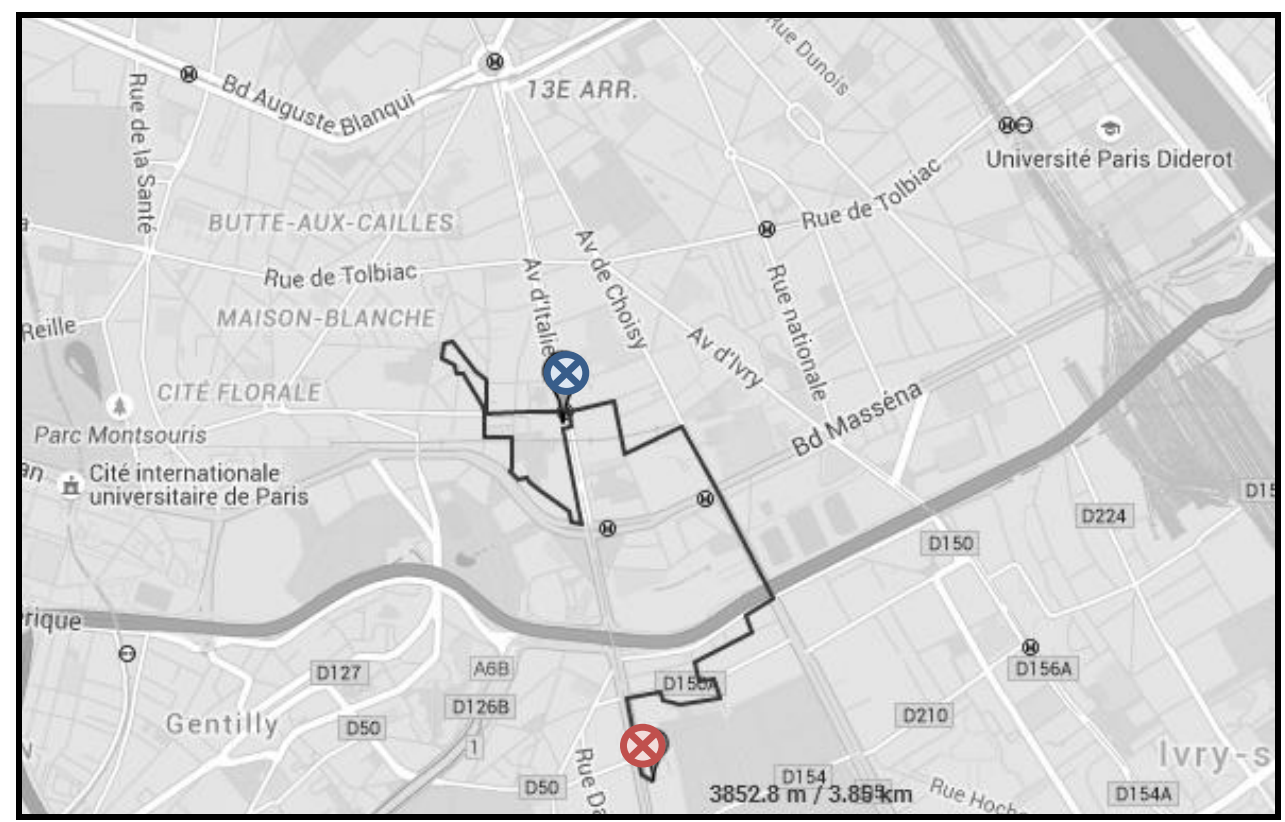

Key:

Q: Starting point, Avenue d'Italie, $11.00 \mathrm{pm}$

8 : Finishing point, shopping mall of the Kremlin Bicêtre, 01.00 am

Figure 4: EUNW D, Paris, 09/17/2015, authors 


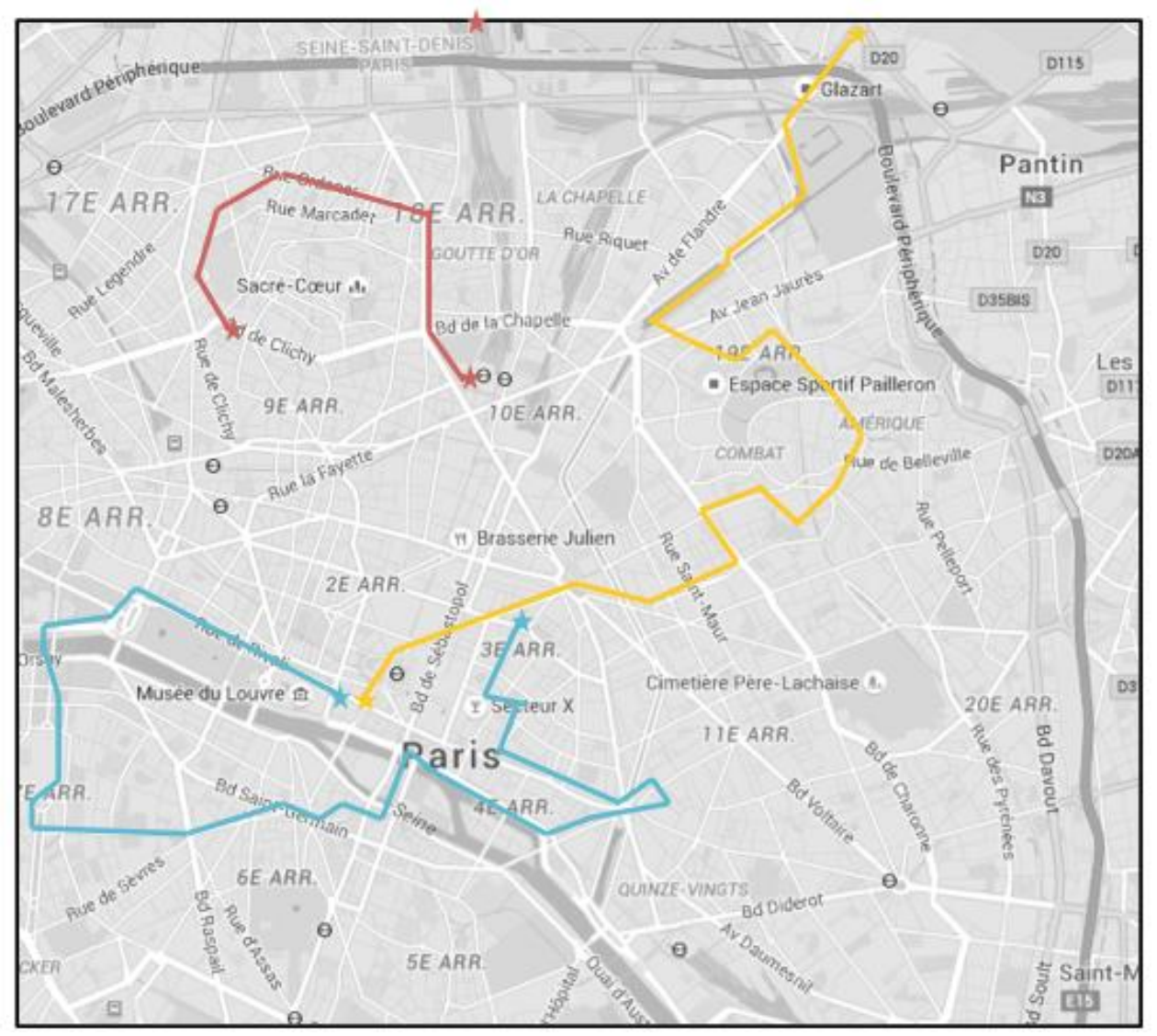

Key:

Yellow team: from Porte de Pantin (11.00 pm) to Place du Châtelet (05.00 am)

: Red team: from Saint-Denis $(10.30 \mathrm{pm})$ to Place du Châtelet $(05.00 \mathrm{am})$

Blue team: from Carreau du Temple (11.00 pm) to Place de Clichy $(04.00 \mathrm{am})$.

\section{Annexe $n^{\circ} 2$, Carnet d'exploration :}

Il s'agit des outils méthodologiques utilisés pour la dernière marche urbaine exploratoire de nuit, la plus aboutie. 


\section{Grille d'observation fixe}

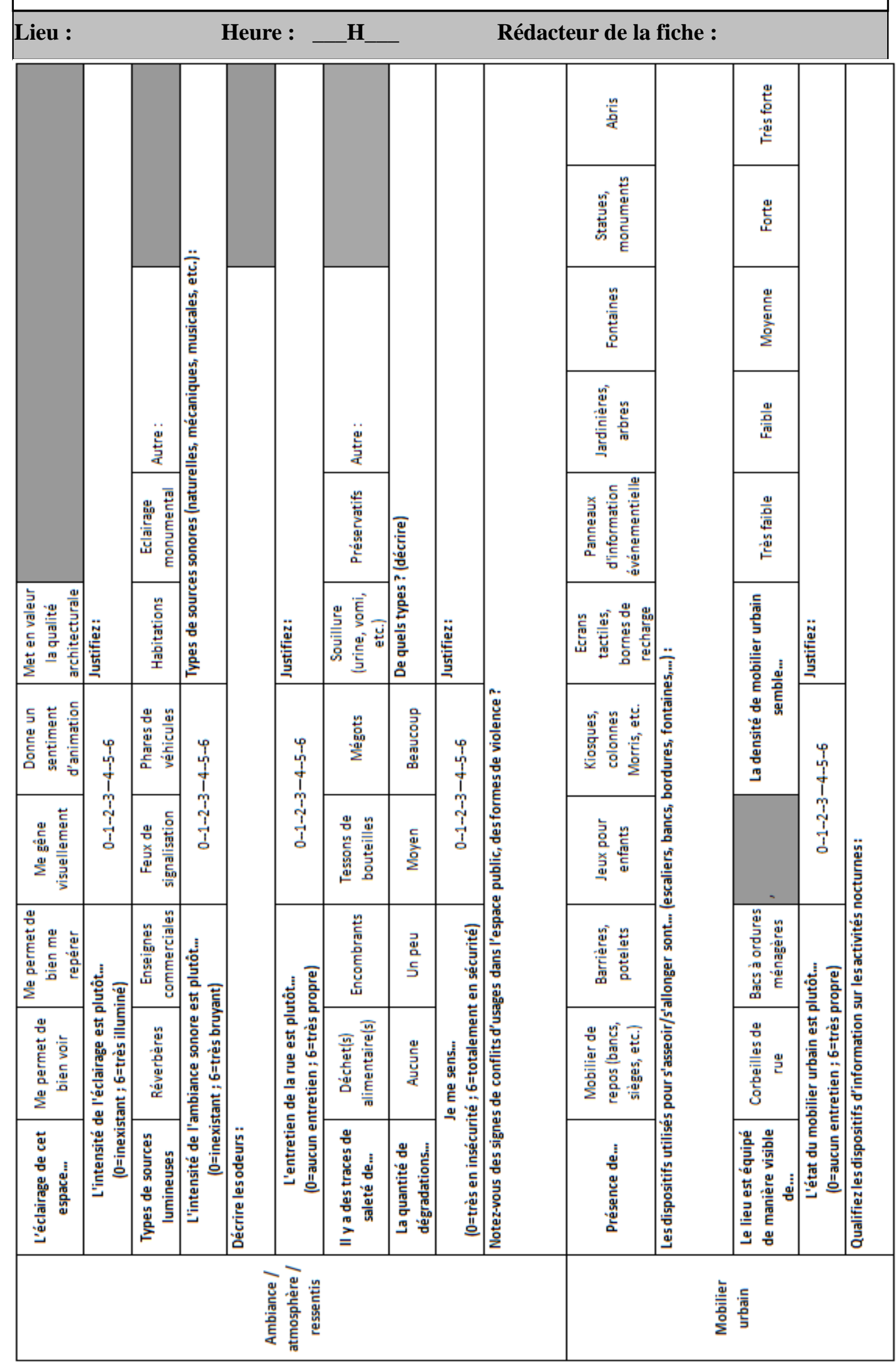




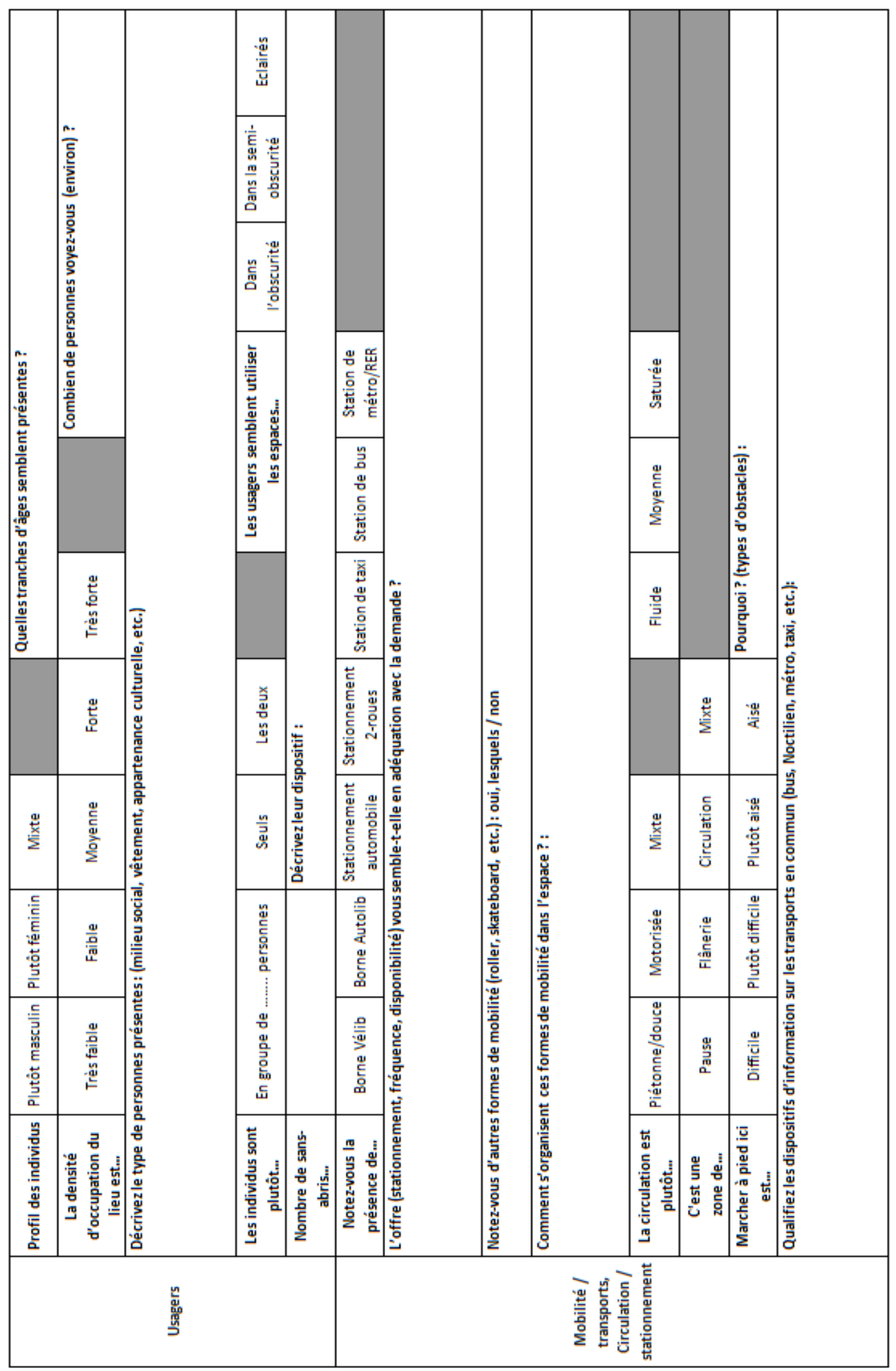

$25 / 32$ 


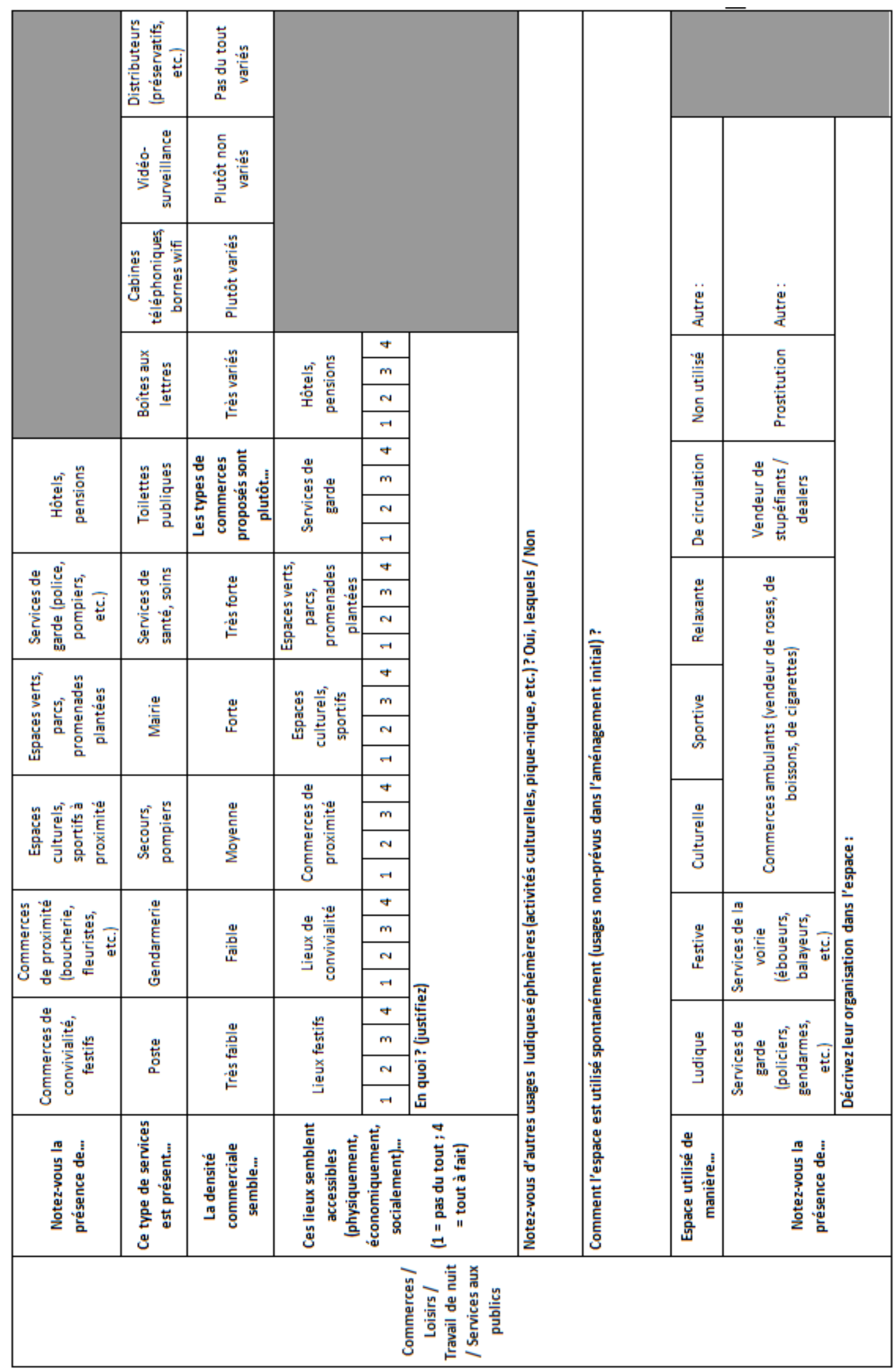




\begin{tabular}{|c|c|c|c|c|}
\hline \multicolumn{2}{|c|}{ Grille d'observation flottante } & Lieu : & \multicolumn{2}{|c|}{ Heure de début : ___ Heure de fin : ___ } \\
\hline Thèmes & & Points positifs & Points négatifs & Opportunités et limites des usages \\
\hline \multicolumn{5}{|l|}{$\begin{array}{l}\text { Propreté (assainissement, } \\
\text { poubelles, encombrants) }\end{array}$} \\
\hline \multicolumn{5}{|l|}{$\begin{array}{c}\text { Sécurité } \\
\text { (éclairage public, caméras } \\
\text { de surveillance, agents de } \\
\text { sécurité...) }\end{array}$} \\
\hline \multicolumn{5}{|l|}{$\begin{array}{l}\text { Aménagement de la } \\
\text { voirie (mabilier urbain, } \\
\text { bancs, marches etc.) }\end{array}$} \\
\hline \multicolumn{5}{|l|}{$\begin{array}{l}\text { Ambiance urbaine } \\
\text { (conviviaitite, animation des } \\
\text { lieux / signes tensian, } \\
\text { vandalisme) }\end{array}$} \\
\hline \multicolumn{5}{|l|}{$\begin{array}{l}\text { Transports publics } \\
\text { (stations kélib, Avetaulib. } \\
\text { arrêts de bus...) }\end{array}$} \\
\hline \multicolumn{5}{|l|}{$\begin{array}{l}\text { Information sur la vie } \\
\text { nocturne / signalétique } \\
\text { (kiosques, panneoux...) }\end{array}$} \\
\hline Usagers/fréquentation & $\begin{array}{l}\text { 1. Hommes } 2 \text {. } \\
\text { 4. Seuls 5. En } \\
\text { Tranches d'â: }\end{array}$ & $\begin{array}{l}\text { Femmes 3. Mixte } \\
\text { groupe de __ personnes } \\
\text { es présentes: }\end{array}$ & Densité d'occupation : & $\begin{array}{l}\text { Type de personnes présentes: } \\
\text { (milieu social, vêtement, appartenance } \\
\text { culturelle, etc.) }\end{array}$ \\
\hline $\begin{array}{l}\text { Comment vous sentez- } \\
\text { vous? }\end{array}$ & Décrivez les 0 & deurs : & Décrivez l’ambiance sonore : & Commentaires \\
\hline
\end{tabular}

\section{Interview noctambule}

\section{Lieu : \\ Heure :}

1/ Trajectoire festive : "Nous allons vous poser des questions sur le déroulement de votre soirée actuelle »

a) Veuillez nous raconter comment a débuté votre soirée :

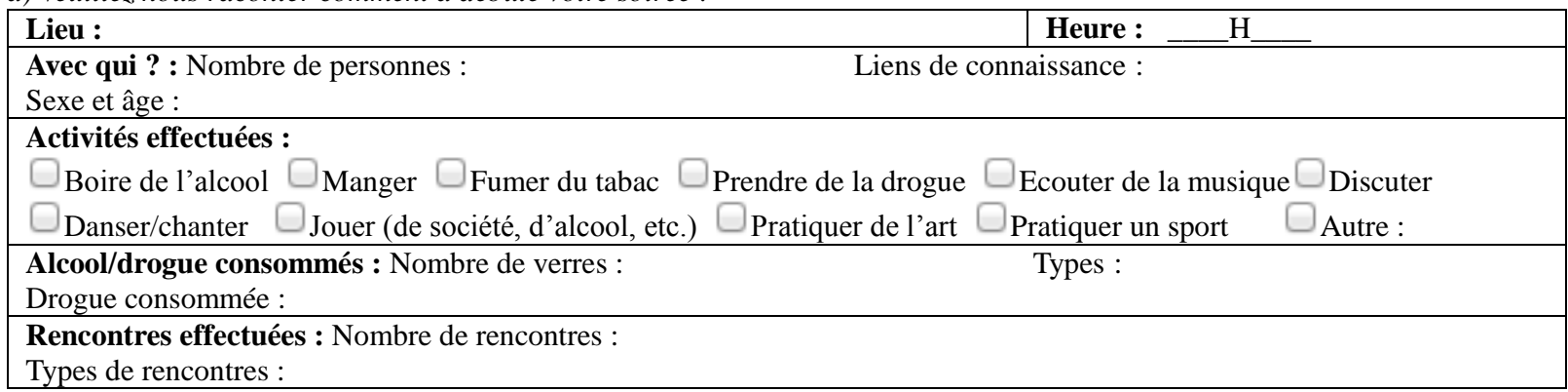

b) Veuillez nous raconter la suite de votre soirée :

\begin{tabular}{|c|c|}
\hline Second lieu : & Heure : \\
\hline $\begin{array}{l}\text { Transport pour s'y rendre : } \\
\square_{\text {A pied }} \square_{\text {En 2-roues }} \square_{\text {En bus }} \square_{\text {En métro/RER }} \\
\square_{\text {En taxi }} \square_{\text {En voiture }} \square_{\text {Autre : }}\end{array}$ & Activités durant ce trajet : \\
\hline $\begin{array}{l}\text { Avec qui ? : Nombre de personnes: } \\
\text { Sexe et âge : }\end{array}$ & Liens de connaissance: \\
\hline $\begin{array}{l}\text { Activités effectuées : } \\
\square_{\text {Boire de l'alcool }} \square_{\text {Manger }} \square_{\text {Fumer du tabac }} \\
\square \text { Danser/chanter } \square \text { Jouer (de société, d'alcool, etc.) }\end{array}$ & $\begin{array}{l}\text { Idre de la drogue } \square_{\text {Ecouter de la musique }} \square_{\text {Discuter }} \\
\text { ratiquer de l'art } \square_{\text {Pratiquer un sport } \quad \square \text { Autre : }}\end{array}$ \\
\hline $\begin{array}{l}\text { Alcool/drogue consommés : Nombre de verres : } \\
\text { Drogue consommée : }\end{array}$ & Types: \\
\hline
\end{tabular}


Rencontres effectuées : Nombre de rencontres :

Types de rencontres :

Qu'est-ce qui vous a motivé à aller dans ces divers lieux?

Quel a été le montant de vos dépenses durant cette soirée jusqu'à maintenant? :
0-10 euros
11-20 euros
21-30 euros
$31-$

50 euros

51-100 euros

101-500 euros

Plus de 500 euros

Quels sont vos plans pour continuer la soirée?

\section{2/ Habitudes de sorties :}

Durant les trois dernières soirées, dans quels types de lieux êtes-vous sortis la nuit à Paris au moins une fois

\begin{tabular}{|c|c|c|c|c|c|c|c|}
\hline $\begin{array}{c}\text { Lieux marchands / } \\
\text { commerciaux : }\end{array}$ & Bars & $\begin{array}{c}\text { Boîtes } \\
\text { de nuit / } \\
\text { clubs }\end{array}$ & $\begin{array}{c}\text { Restaurants } \\
\text { / brasseries }\end{array}$ & Fast-food & $\begin{array}{c}\text { Salles de } \\
\text { spectacle }\end{array}$ & Cinémas & $\begin{array}{c}\text { Sex- } \\
\text { clubs/sex- } \\
\text { shops }\end{array}$ \\
\hline $\begin{array}{c}\text { Lieux non- } \\
\text { commerciaux : }\end{array}$ & Squats & $\begin{array}{c}\text { Rues, } \\
\text { places }\end{array}$ & $\begin{array}{c}\text { Parcs, } \\
\text { espaces } \\
\text { verts }\end{array}$ & $\begin{array}{c}\text { Berges de } \\
\text { Seine, de } \\
\text { canal }\end{array}$ & $\begin{array}{c}\text { Soirée en } \\
\text { lieu privé }\end{array}$ & \\
\hline Événements festifs : & \multicolumn{2}{|c|}{$\begin{array}{c}\text { Institutionnels } \\
\text { (Nuits Blanches, } \\
\text { etc.) }\end{array}$} & $\begin{array}{c}\text { Alternatifs, informels } \\
\text { (Friches industrielles, } \\
\text { etc.) }\end{array}$ & $\begin{array}{c}\text { Soirées éphémères et } \\
\text { payantes }\end{array}$ & $\begin{array}{c}\text { Fêtes municipales, } \\
\text { nationales }\end{array}$ \\
\hline
\end{tabular}

Durant les trois dernières soirées, dans quels quartiers parisiens êtes-vous sortis la nuit?

Pour ces trois dernières soirées, quels jours de la semaine êtes-vous sortis?

$\square_{\text {Lundi }} \square_{\text {Mardi }} \square_{\text {Mercredi }} \square_{\text {Jeudi }} \square_{\text {Vendredi }} \square_{\text {Samedi }} \square_{\text {Dimanche }}$

Pour ces trois dernières soirées, quels ont été vos horaires de sortie approximatifs?

Soirée 1:__

$\overline{\mathrm{Habituellement}}$, quelle est la fréquence de vos sorties à Paris la nuit ?

Tous les jours

1 fois par semaine
4 à 6 fois par semaine

Plusieurs fois par mois
2 à 4 fois par semaine

1 fois par mois

Moins souvent

3/Ambiances de la rue :

L'ambiance de cette rue vous semble... ( $(0=$ très désagréable $; 6=$ très agréable $)$

L'éclairage de cette rue est... ( $0=$ très sombre ; $6=$ très éclairée $)$

L'ambiance sonore de cette rue est $\ldots(0=$ très calme $; 6=$ très bruyante $)$

L'entretien de cette rue est... ( $0=$ aucun entretien ; $6=$ très propre $)$

Je me sens... ( $0=$ en totale insécurité ; $6=$ totalement en sécurité)

$0--1--2--3-4--5--6$ $0--1--2--3-4--5--6$

$0--1--2--3-4--5--6$

$0--1--2--3-4--5--6$

Que pensez-vous de la propreté de la rue et de son aménagement par rapport à vos usages nocturnes?

En quoi vous sentez-vous plus ou moins libres / contraints dans l'espace public à Paris, la nuit? 
4/ Perception des nuits parisiennes:

Pour qualifier les nuits parisiennes actuelles, $j$ 'utiliserais les trois mots :

$1 /$

$2 /$

$3 /$

Quelles sont les pistes d'amélioration que vous proposeriez pour la vie nocturne de Paris?

Connaissez-vous ces nouvelles mesure/expérimentations mises en place par la Ville pour améliorer la vie nocturne parisienne? :

\begin{tabular}{|l|l|l|}
\hline Mesures & Oui/Non & Si oui, avis : \\
\hline Le Site Que faire à Paris - nuitsdeparis.fr & & \\
\hline $\begin{array}{l}\text { Ouverture nocturne de centres d'animation } \\
(3)\end{array}$ & & \\
\hline $\begin{array}{l}\text { Ouverture nocturne d'équipements sportifs } \\
(17)\end{array}$ & & \\
\hline Ouverture nocturne de parcs et jardins (4) & & \\
\hline Désignation d'un adjoint à la nuit & & \\
\hline $\begin{array}{l}\text { Soutien à 9 festivals électro (Weather } \\
\text { festival...) }\end{array}$ & & \\
\hline
\end{tabular}

Quels sont les meilleurs atouts de la vie nocturne parisienne?

Quels sont les meilleurs atouts de la vie noctume parisienne?

5/Informations sociodémographiques :

Année de naissance :

Sexe: $M F$

Activité professionnelle :

Lieu de résidence (Ville, arrondissement/quartier) :

Situation familiale :

Conditions de vie (salaire, être à l'aise financièrement ou non, etc.)

\section{Interview commerçant / travailleur de nuit}

Lieu : $\quad$ Heure : ___

Fonction :

Type de contrat :

Horaires

de

travail :

1/ Description de l'emploi :

Depuis combien de temps exercez-vous ce métier?

A quelle fréquence travaillez-vous de nuit?

Quels sont les difficultés et les atouts pour exercer cet emploi de nuit?

\begin{tabular}{|l|l}
\hline Difficultés : & Atouts :
\end{tabular} 
Y a-t-il des nouvelles réglementations nocturnes portant sur votre métier? Si oui, comment vous y adaptez-

vous?

2/ Clientèle/usagers du commerce :

Quel est le profil de la clientèle/des usagers nocturnes par rapport à la journée?

- Nombre de clients (par nuit, semaine, mois ou événement) :

- Nombre/pourcentage de touristes :

- $\quad$ Sexe et âge des clients :

- Milieu social, culturel des clients :

- Plutôt des habitués, nouveaux clients ? :

Que pensez-vous de l'évolution de la clientèle/des usagers nocturnes ces dernières années, s'il y a eu évolution?

Quelles sont vos relations avec le voisinage? Cela vous arrive-t-il d'intervenir auprès des usagers pour gérer des conflits, nuisances, etc. ? (Anecdote?)

\section{3/ Perception de la vie nocturne :}

Comment a évolué la vie nocturne dans ce quartier?

L'ambiance globale des nuits parisiennes vous semble ... $(0=$ très désagréable ; $6=$ très agréable $)$ ? 0 --1---2---3-4---5-6

Pour qualifier les nuits parisiennes actuelles, vous utiliseriez les trois mots :

$1 /$ $2 /$

Quels types d'attentes avez-vous par rapport à la vie nocturne de Paris?

Quels sont les meilleurs atouts de la vie nocturne parisienne? 
Quelles pistes d'amélioration concrètes proposez-vous pour la vie nocturne parisienne (meilleure utilisation du mobilier urbain, autre type d'éclairage urbain, etc.) ?

\section{4/Informations sociodémographiques :}

Année de naissance :

Lieu de résidence (Ville, arrondissement/quartier) :

temps?:

Situation familiale :

Conditions de vie (salaire, être à l'aise financièrement ou non, etc.) :

"Etes-vous d'accord pour être contacté par la suite dans le cadre de notre étude?

Email :

Tél :
Sexe: $M F$

Depuis combien de

\section{Carte mentale}

Nous souhaiterions que vous dessiniez une carte de votre parcours nocturne. Vous devez nous montrer la manière dont vous avez vécu cette expérience. Essayez de dessiner tous les éléments principaux. Nous n'attendons pas un dessin professionnel, simplement de voir ce dont vous vous souvenez. Ce n'est pas important si vous ne vous rappelez pas des noms des lieux. Dessinez ce que vous aimez ou non, un sentiment, un objet, .... Ce n'est pas un test de mémoire ou de compétence, mais, une autre manière de partager votre expérience vécue de la traversée.

\section{Feuille A4}


Commentez votre carte mentale :

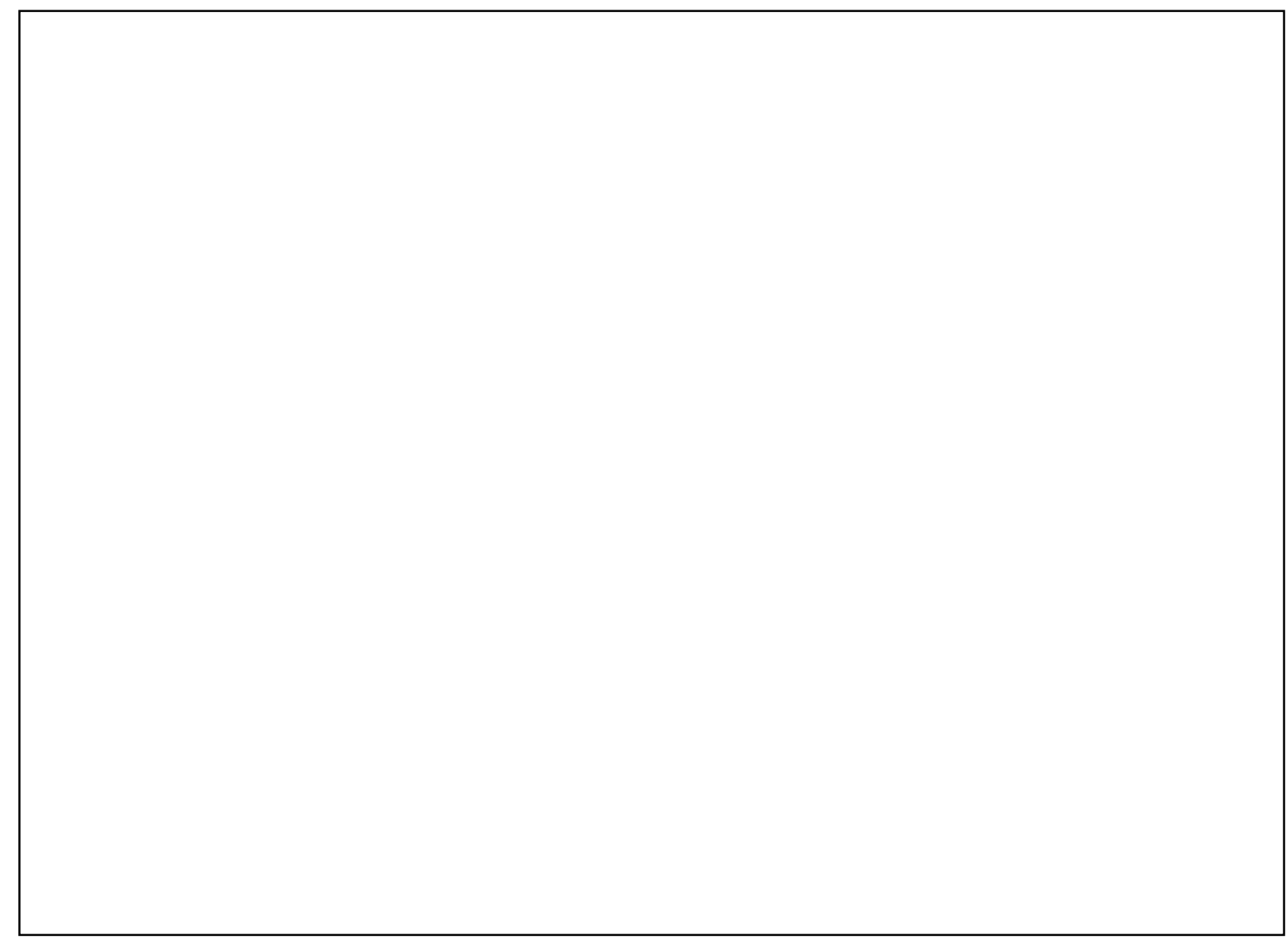

